\title{
(A)symmetries of weak decays at and near the kinematic endpoint
}

\author{
Gudrun Hiller $^{a}$ and Roman Zwicky ${ }^{b}$ \\ ${ }^{a}$ Institut für Physik, Technische Universität Dortmund, \\ D-44221 Dortmund, Germany \\ ${ }^{b}$ School of Physics \& Astronomy, University of Edinburgh, \\ Edinburgh EH9 3JZ, Scotland \\ E-mail: ghiller@physik.uni-dortmund.de, Roman.Zwicky@ed.ac.uk
}

ABSTRACT: At the kinematic endpoint of zero recoil physical momenta are parallel which leads to symmetries in the decay distributions. We implement this observation for decays of the type $A \rightarrow\left(B_{1} B_{2}\right) C$ by extending the helicity formalism to include an unphysical timelike polarisation. The symmetries of the helicity amplitudes are worked out for a generic dimension six Hamiltonian for a $B \rightarrow V \ell \ell$ decay type. We obtain exact predictions for angular observables, e.g., for the fraction of longitudinally polarized vector mesons, $F_{L}=1 / 3$, which may be used to guide experimental analyses. We investigate the vicinity of the endpoint through an expansion in the three momentum of the vector meson. New physics can be searched for in the slope of the observables near the endpoint. Current experimental data on $B \rightarrow K^{*} \ell \ell$ decays are found to be in agreement with our predictions within uncertainties. Application to other semileptonic $B$ and $D$ decays, including $B \rightarrow$ $V \ell^{+} \ell^{-}, V=K^{*}, \phi, \rho$ and $B \rightarrow V \ell \nu, V=\rho, D^{*}$ is straightforward. For hadronic modes of the types $B \rightarrow V p \bar{p}, V \Lambda \bar{\Lambda}, \ldots$ and $B \rightarrow V \pi \pi, V \pi K, \ldots$ endpoint relations apply as long as they are not overwhelmed by sizeable final state interactions between the $V$ and the hadron pair.

Keywords: Rare Decays, B-Physics

ARXIV EPRINT: 1312.1923 


\section{Contents}

1 Introduction 1

2 Endpoint relations for decays induced by effective Hamiltonians $\quad 2$

2.1 The decay $A \rightarrow\left(B_{1} B_{2}\right) C$ in terms of generalised helicity amplitudes 2

2.2 The tensor operator transition for $B \rightarrow K^{*} \ell \ell \quad 4$

2.3 Summary of endpoint relations for $B(D) \rightarrow V \ell \ell \quad 5$

2.4 Parity selection rules the transversity amplitudes 6

3 Semileptonic $B$ and $\boldsymbol{D}$ decays into vector mesons 8

3.1 Kinematic endpoint: observables in a general dimension six operator basis $\quad 9$

3.2 Small momentum expansion (low recoil): the SM + SM' basis 10

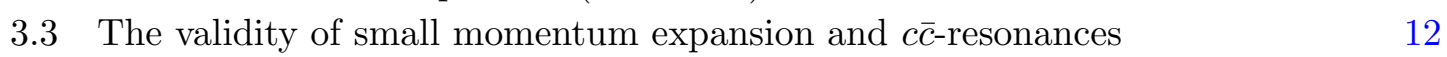

$\begin{array}{lll}\text { 3.3.1 The (non-)impact of the } c c \text {-resonances at low recoil } & 12\end{array}$

3.3.2 The small momentum expansion at work for $F_{L}$ and $A_{T}^{(2)} \quad 13$

$\begin{array}{ll}\text { 3.3.3 Low recoil OPE versus non-factorizable corrections } & 14\end{array}$

$\begin{array}{ll}3.4 & \text { Applications to other vector modes } \\ \end{array}$

$4 \quad B \rightarrow X_{j} \ell \ell$-type decays $\quad 16$

$\begin{array}{llr}4.1 & \text { Spin } 0 & 16\end{array}$

$\begin{array}{lll}4.2 & \text { Higher spin } & 17\end{array}$

5 Endpoint relations and non-leptonic decays $\quad \mathbf{1 8}$

$\begin{array}{ll}5.1 \text { Hadronic } S \rightarrow V_{1} V_{1} \text { and qualitative remarks on } F_{L} & 19\end{array}$

$\begin{array}{llr}6 & \text { Summary } & 20\end{array}$

$\begin{array}{ll}\text { A Conventions for polarisation vectors } & 22\end{array}$

B The low recoil OPE and non-factorizable corrections in the light of end$\begin{array}{ll}\text { point relations } & 23\end{array}$

C Phenomenological parameterisations for helicity amplitudes $\quad 24$

$\begin{array}{lll}\text { C.1 Low recoil - high } q^{2} & 25\end{array}$

C.2 Large recoil — low $q^{2} \quad 26$

$\begin{array}{lll}\text { C.3 Three momentum } \kappa \text { versus } q^{2} & 26\end{array}$

$\begin{array}{lll}\text { D } F_{L} \text { asymptotics } & 27\end{array}$

$\begin{array}{lll}\mathrm{E} & \text { Tensor contributions to } d \Gamma / d \phi \text { at the endpoint } & 27\end{array}$ 


\section{Introduction}

At the kinematic endpoint of a decay the relevant spatial momenta are zero and, in the absence of initial state polarization, there is no preferred axis. This leads to isotropicity in certain observables. At the formal level it implies a reduction in the number of independent Lorentz invariants. We implement this idea for decays of the type $A \rightarrow\left(B_{1} B_{2}\right) C\left(\rightarrow C_{1} C_{2}\right)^{1}$ for which the kinematic endpoint, in the $A$-restframe, is defined by $\vec{q}_{C}=0$ and hence $\vec{q}_{B_{1}}+\vec{q}_{B_{2}}=0$. In the discussion it is implicit that the decay products of the $C$-particle do not interact significantly with the $\left(B_{1} B_{2}\right)$-pair. In the case where the $\left(B_{1} B_{2}\right)$-pair originates from a particle, denoted by $B$ in figure 1 (left), the helicity formalism [1] (and [2] for a review) provides a powerful tool for the description of the kinematic endpoint [3]. The goal of this work is to extend this idea to the case where the decay is described, in part, by an interaction of the type $H^{\mathrm{eff}} \propto A^{\dagger} C\left(B_{1} B_{2}\right)$ as depicted in figure 1 (right). This case is still amenable to a helicity formalism provided one introduces an unphysical timelike polarisation direction, see section 2 .

Concrete examples arise in flavor physics, where semileptonic decays of beauty and charm mesons are described by a $|\Delta F|=1$ effective Hamiltonian. The latter contain terms e.g., $\mathcal{H}_{b \rightarrow s \ell \ell}^{\text {eff }} \propto \bar{s}_{L} \gamma_{\mu} b \bar{\ell} \gamma^{\mu} \gamma_{5} \ell+\ldots$ originating from integrating out $Z$ - and $W$-bosons which cannot be interpreted as originating from a sequential decay of the type $B \rightarrow K^{*} \gamma^{*}(\rightarrow \ell \ell)$. Predictions for angular observables at the endpoint are worked out for a large class of decays in section 3 ; here, $B \rightarrow K^{*} \ell \ell$ serves as a template.

It is noteworthy that the endpoint relations have to emerge in any consistent approximation that respects Lorentz invariance. The low recoil operator product expansion (OPE) [4] for $B \rightarrow K^{*} \ell \ell$ is one such example. Here, after employing the improved IsgurWise relations $[5,6]$ the endpoint symmetries are manifest [7]. For recent applications, see e.g., $[8,9]$. We emphasise that endpoint relations are not directly related to Isgur-Wise relations although both emerge at small or zero velocities. Endpoint relations are of purely kinematic nature and relate helicity amplitudes of different directions. The Isgur-Wise relations are based on QCD equation of motions and relate tensor and vector currents of the same helicity direction. In the context of the aforementioned low recoil OPE they readily serve to implement the heavy quark expansion on the level of matrix elements.

The exactness of the endpoint relations raises the question of the behaviour in the vicinity of the endpoint-region. We investigate this question through an expansion in the magnitude of the three-momentum $\kappa=\left|\overrightarrow{q_{C}}\right|$ of the $C$-particle in the $A$-restframe.

The endpoint relations hold for the reconstructed decay in the endpoint configuration where $\vec{q}_{C}=\vec{q}_{B_{1}}+\vec{q}_{B_{2}}=0$. Being based on kinematics the relations are independent of the production mechanism and prevail, as previously mentioned, provided the $C$-particle's decay products do not significantly interact with the $\left(B_{1} B_{2}\right)$-pair. For the $B \rightarrow K^{*} \ell \ell$-type decay this is the case as interactions are of the electroweak type and therefore negligible. For non-leptonic decays this is less evident as there can be inelasticities from rescattering into different final states. As discussed in section 5 this results in only a subset of events

\footnotetext{
${ }^{1}$ The extension to $A \rightarrow\left(B_{1} B_{2} \ldots\right) C\left(\rightarrow C_{1} C_{2} \ldots\right)$-type decays is straightforward although the multi-body angular distributions will grow in complexity.
} 

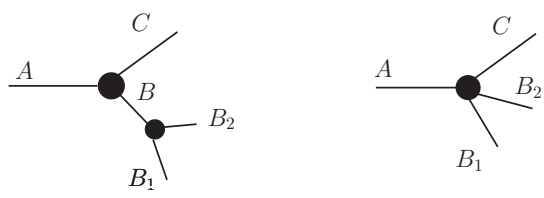

Figure 1. Decay $A \rightarrow B\left(\rightarrow B_{1} B_{2}\right) C$ with intermediate $B$-particle which is generically off-shell (left). Decay $A \rightarrow\left(B_{1} B_{2}\right) C$ described by a point interaction (right). The endpoint configuration is described in the $A$-restframe by $\vec{q}_{C}=0$ and implies that $B_{1}$ and $B_{2}$ are back-to-back. Possible decay products of the $C$-particle should have sufficiently suppressed interactions with the $\left(B_{1} B_{2}\right)$-pair for the endpoint relation to remain visible in the decay.

remaining within the endpoint configuration. Furthermore, the relations do not apply in the case of missing momentum, e.g., a soft photon being unnoticed in the detector. The process is then, however, a different one $A \rightarrow\left(B_{1} B_{2}\right) C \gamma$ at the price of shifting the maximum $\left(B_{1} B_{2}\right)$-invariant mass to lower values depending on the photons' energy.

The paper is organised as follows: in section 2 we discuss the helicity formalism in the effective Hamiltonian formulation. In section 3 we work out the implications of our findings for angular decay observables for $B \rightarrow V \ell \ell$-type decays in the generic dimension six effective Hamiltonian. Sections 3.2 and 3.3 contain most of the phenomenological results. We discuss $B \rightarrow K \ell \ell$-type decays as well as higher spin final states in section 4 . To what extent endpoint relations remain valid for non-leptonic decays is investigated in section 5. In section 6 we summarise. Useful formulae for polarisation vectors are compiled in appendix A. The discussion of the low recoil OPE for $B \rightarrow K^{(*)} \ell \ell[4]$ from the viewpoint of endpoint symmetries is deferred to appendix B. Parameterisations of helicity amplitudes and considerations on asymptotic values of the fraction of longitudinal polarization are given in appendix C and D, respectively. Details on the tensor contributions at the endpoint can be seen in appendix E.

\section{Endpoint relations for decays induced by effective Hamiltonians}

We discuss the reduction of Lorentz-invariants in terms of helicity amplitudes (HAs) at the kinematic endpoint for $A \rightarrow\left(B_{1} B_{2}\right) C$ type decays. Below we show using a generic language that such decays are amenable to a HA treatment provided one introduces a timelike polarisation reminiscent of covariant (Gupta-Bleuler) quantization of a massive spin 1 boson.

\subsection{The decay $A \rightarrow\left(B_{1} B_{2}\right) C$ in terms of generalised helicity amplitudes}

The amplitude for a $A \rightarrow\left(B_{1} B_{2}\right) C$ decay can be written in factorized form

$$
\mathcal{A}\left(A \rightarrow\left(B_{1} B_{2}\right) C\right)=\left(B_{1} B_{2}\right)_{\mu_{1} \ldots \mu_{X}} C^{\mu_{1} \ldots \mu_{X}}\left(q_{A}^{2}, q_{B}^{2}, q_{C}^{2}\right) .
$$

The symbols $q_{r}, \lambda_{r}$, and $m_{r}, r=A, B, C$ denote the corresponding four momenta, helicities and masses, respectively, where $q_{B}=q_{B_{1}}+q_{B_{2}}$. In the notation above we have not imposed the on shell conditions $q_{r}^{2}=m_{r}^{2}$. The crucial point in eq. (2.1) is that the momenta of $q_{B_{1,2}}$ 
do not enter the dynamics as otherwise six invariants of a $1 \rightarrow 3$ decay would govern the form factor $C$ above. Contributions from $A \rightarrow\left(B^{*} \rightarrow B_{1} B_{2}\right) C$, with $B$ being potentially off-shell, are a special and simple case of eq. (2.1). On a formal level interactions of the type in eq. (2.1) are still amenable to a helicity-like treatment provided one introduces a fourth (unphysical) helicity direction $\omega\left(t, q_{r}\right) \propto\left(q_{r}\right)$ ( $t$ stands for timelike). Some more details and remarks on conventions can be found in appendix A. This allows for the following completeness relation

$$
\sum_{\lambda, \lambda^{\prime} \in\{t, \pm, 0\}} \omega^{\mu}(\lambda) \omega^{* \nu}\left(\lambda^{\prime}\right) G_{\lambda \lambda^{\prime}}=g^{\mu \nu}, \quad G_{\lambda \lambda^{\prime}}=\operatorname{diag}(1,-1,-1,-1),
$$

where the first entry in $G_{\lambda \lambda^{\prime}}$ refers to $\lambda=\lambda^{\prime}=t$. Relation (2.2) can be inserted $X$-times to obtain a HA with $X$ helicity indices in addition to the ones from the $A$ and $C$ particles. In the notation of (2.1) the amplitude may be written in terms of (generalised) helicity amplitude as follows,

$$
\begin{aligned}
& \mathcal{A}\left(A \rightarrow\left(B_{1} B_{2}\right) C\right)=H_{\lambda_{B_{1}} \ldots \lambda_{B_{X}}, \lambda_{C}}\left(B_{1} B_{2}\right)_{\lambda_{B_{1}^{\prime}} \ldots \lambda_{B_{X}^{\prime}}} G^{\lambda_{B_{1}} \lambda_{B_{1}^{\prime}}} \ldots G^{\lambda_{B_{X}} \lambda_{B_{X}^{\prime}}}, \\
& \left(B_{1} B_{2}\right)_{\lambda_{B_{1}^{\prime}} \ldots \lambda_{B_{X}^{\prime}}}=\left(B_{1} B_{2}\right)^{\mu_{1} \ldots \mu_{X}}\left[\beta_{\mu_{1}}\left(\lambda_{B_{1}}\right) \ldots \beta_{\mu_{X}}\left(\lambda_{B_{X}}\right)\right] \text {, } \\
& H_{\lambda_{B_{1}} \ldots \lambda_{B_{X}}, \lambda_{C}}=C^{\alpha \mu_{1} \ldots \mu_{X}} \gamma_{\alpha}^{*}\left(\lambda_{C}\right)\left[\beta_{\mu_{1}}^{*}\left(\lambda_{B_{1}}\right) \ldots \beta_{\mu_{X}}^{*}\left(\lambda_{B_{X}}\right)\right],
\end{aligned}
$$

with $J_{A}=0$ and $J_{C}=1$ for the sake of illustration. In the first equation we have implied the Einstein summation convention for the helicity indices. Formally eq. (2.3) may be interpreted as a decay with $X$ intermediate vector bosons which can take on physical as well as timelike polarisation directions. In the two-body decay $A\left(\lambda_{A}\right) \rightarrow B\left(\lambda_{B}\right) C\left(\lambda_{C}\right)$ where the helicity quantization axis is taken to be the decay axis angular momentum conservation implies $\lambda_{A}=\lambda_{B}-\lambda_{C}$. More precisely it is the azimuthal symmetry around the decay axis that gives rise to this result. This can be used to define a generalised helicity conservation $(G H C)$ rule: generically a polarisation vector $\zeta(\lambda, l)$ transforms under a rotation around the $\vec{l}$-axis by the azimuthal angle $\phi$ as follows,

$$
\zeta(t) \rightarrow \zeta(t), \quad \zeta(0) \rightarrow \zeta(0), \quad \zeta( \pm 1) \rightarrow e^{\mp i \phi} \zeta( \pm 1)
$$

since $\sqrt{2} \vec{\zeta}_{ \pm}=\vec{e}_{1} \pm i \vec{e}_{2}$ where $\vec{e}_{1}$ and $\vec{e}_{2}$ are unit vectors orthogonal to $\vec{l}$. The polarisation vectors $\alpha\left(\lambda, q_{A}\right), \beta\left(\lambda, q_{B}\right)$ and $\gamma\left(\lambda, q_{C}\right)^{*}=\gamma\left(\bar{\lambda}, q_{C}\right)$, where $\bar{\lambda} \equiv-\lambda$ throughout, transform identically. Hence the GHC rule reads as follows:

$$
\lambda_{A}=\sum_{i=1}^{X} m\left(\lambda_{B_{i}}\right)+\bar{\lambda}_{C}, \quad m(t)=m(0)=0, m( \pm 1)= \pm 1 .
$$

Below we shall omit the function $m$ for brevity. Since $\lambda_{A}$ is fixed by eq. (2.5) it can be omitted from the labels in (2.3). Moreover, in the case where $\lambda_{A}=0$, as happens in the case of $B$ and $D$ meson decays, $\lambda_{C}=\sum \lambda_{B_{i}}$ and it is therefore possible and customary to omit $\lambda_{C}$ as well:

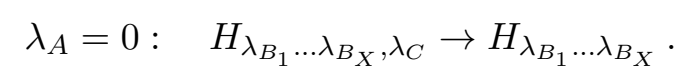


In the remainder of this section we discuss the endpoint symmetries of the HAs that enter the most generic dimension six $b \rightarrow$ sll effective Hamiltonian. The basis consists of 10 operators given in section 2.3 together with a summary of endpoint relations. We discuss the tensor operator, which is the most complicated case to analyze, in the next section.

\subsection{The tensor operator transition for $B \rightarrow K^{*} \ell \ell$}

We study tensor operators in $b \rightarrow$ sll transitions:

$$
H_{\mathrm{eff}} \propto O_{\mathcal{T}}+\ldots, \quad O_{\mathcal{T}} \equiv \bar{s}_{L} \sigma^{\mu \nu} b \bar{\ell} \sigma_{\mu \nu} \ell
$$

In the notation of eq. (2.1) $X=2$ and $\left(B_{1} B_{2}\right)_{\mu_{1} \mu_{2}}=\bar{\ell} \sigma_{\mu \nu}\left(\gamma_{5}\right) \ell$. The $\sigma$-Dirac structure corresponds to the antisymmetric spin 1 representation (in $\mathrm{SO}(3)$ notation: $(\mathbf{3} \times \mathbf{3})_{\mathrm{SO}(\mathbf{3})}=$ $\mathbf{3}_{\mathbf{A}}+\ldots$ where $A$ stands for antisymmetric and we denote the representations by $\mathbf{l}$ rather than its dimension $\operatorname{dim}(\mathbf{l})=2 l+1)$. We combine the two polarisation vectors into an antisymmetric "helicity"-tensor

$$
\beta_{\mu \nu} \equiv \beta_{\mu}\left(\lambda_{B_{1}}\right) \beta_{\nu}\left(\lambda_{B_{2}}\right)-\{\mu \leftrightarrow \nu\}
$$

not to be confused with a proper spin 2 helicity tensor. Using GHC (2.5) we identify two classes of potentially non-vanishing HAs,

$$
X_{1}=\left\{H_{t 0}, H_{t+}, H_{t-}\right\}, \quad X_{2}=\left\{H_{+-}, H_{+0}, H_{-0}\right\} .
$$

Above we have used the notation (2.6). The helicity indices of the vector meson are 0,1 and -1 in increasing order in the brackets.

For $B \rightarrow K^{*} \ell \ell$ it is customary to denote by $p$ and $q$ the four momentum of the vector meson and the lepton pair $\left(q_{B}=q\right.$ and $q_{C}=p$ in the notation (2.1)). The HAs can be written in terms of Lorentz invariants $P_{i}$ as follows:

$$
H_{\lambda_{B_{1}} \ldots \lambda_{B_{X}}}=\sum_{i=1}^{N} a_{i}\left(q^{2}\right) P_{i}\left(\beta\left(\lambda_{B_{1}}\right), \ldots, \beta\left(\lambda_{B_{X}}\right), \gamma\left(\lambda_{C}\right), q, p\right),
$$

with $N$ finite at a certain order of effective Hamiltonian. We have omitted $p^{2}=m_{V}^{2}$ and $(q+p)^{2}=m_{B}^{2}$ from the form factor as the corresponding particles are on shell. At the endpoint we have got $p \propto q \propto(1,0,0,0) \equiv \omega(t)$, leading to a reduction in the number of invariants $N_{e} \leq N$ where the subscript $e$ stands for endpoint. In the case of sequential decays, that is without timelike polarisation $N_{e} \leq 1$, independent of the effective Hamiltonian [3]. We find $N=2$ for the tensor transition in two ways: $i$ ) writing down possible invariants and ii) using simple arguments of representation theory with Clebsch Gordan coefficients (CGC).

i) Through invariants:

at the endpoint $p \propto q$ implies $\omega(\lambda)=\beta(\lambda, q)=\gamma(\bar{\lambda}, p)$. In attempting to build the number of invariants $N_{e}$ in (2.10) we may therefore involve the following covariants 
$\omega_{\mu \nu}^{*}\left(\lambda_{B_{1}}, \lambda_{B_{2}}\right)(2.8) \omega_{\mu}^{*}\left(\bar{\lambda}_{C}\right)$ and $\omega_{\mu}^{*}(t)$. Two types of invariants can be formed:

$$
\begin{aligned}
& \left(P_{1}\right)_{\lambda_{B_{1}} \lambda_{B_{2}}}=\omega^{* \mu \nu}\left(\lambda_{B_{1}}, \lambda_{B_{2}}\right) \omega_{\mu}^{*}(t) \omega_{\nu}^{*}\left(\bar{\lambda}_{C}\right), \\
& \left(P_{2}\right)_{\lambda_{B_{1}} \lambda_{B_{2}}}=\epsilon^{\mu \nu \rho \sigma} \omega_{\mu \nu}^{*}\left(\lambda_{B_{1}}, \lambda_{B_{2}}\right) \omega_{\rho}^{*}(t) \omega_{\sigma}^{*}\left(\bar{\lambda}_{C}\right) .
\end{aligned}
$$

As the notation suggests the identification with (2.9) is as follows: $\left(P_{i}\right)_{\lambda_{B_{1}} \lambda_{B_{2}}} \leftrightarrow$ $X_{i}=\left\{H_{\lambda_{B_{1}} \lambda_{B_{2}}}\right\}$ for $i=1,2$.

ii) Through $C G C$ : first, if only spatial indices are considered then $\omega_{\mu \nu}^{*}$ corresponds to the antisymmetric vector representation $(\mathbf{3} \times \mathbf{3})_{\mathrm{SO}(3)}=\mathbf{3}_{A}+\ldots$ Considering $\mathbf{3}_{A}$ directly corresponds to $P_{2}$ in (2.11). The role of $\omega^{*}(t)$ is to reduce the Lorentz Levi-Cevita tensor to the $\mathrm{SO}(3)$ antisyemtric tensor $\epsilon_{i j k}$ (with $i, j, k=1 \ldots 3$ ). Second if $P_{1}$ is not to vanish, then $\omega_{\mu}(t)$ can only be contracted with itself and does therefore not bring in a new element. Hence the helicity $\lambda_{C}$ equals the helicity of $\lambda_{B_{1}}$ or $\lambda_{B_{2}}$. In terms of CGCs:

$$
\begin{array}{ll}
X_{1}: H_{t \lambda_{B_{2}}} \sim C_{0 \lambda_{B_{2}} \bar{\lambda}_{B_{2}}}^{011} \leftrightarrow\left(\mathbf{3}_{C} \times \mathbf{3}_{B_{1}}\right)=\mathbf{1}+\ldots, \\
X_{2}: H_{\lambda_{B_{1}} \lambda_{B_{2}}} \sim C_{0 \lambda_{C} \bar{\lambda}_{C}}^{011} C_{\lambda_{C} \bar{\lambda}_{B_{1}} \bar{\lambda}_{B_{2}}}^{111} \leftrightarrow\left(\mathbf{3}_{B_{1}} \times \mathbf{3}_{B_{2}}\right)_{A} \times \mathbf{3}_{C}=\mathbf{1}+\ldots,
\end{array}
$$

where we have used the notation $\lambda_{C}=\lambda_{B_{1}}+\lambda_{B_{2}}$ in the second line of (2.12). The notation for the CGC is $C_{M m_{1} m_{2}}^{J j_{1} j_{2}}$ for $j_{1} \times j_{2}=\ldots+J+\ldots$ with $M=m_{1}+m_{2}$. In the equation above $\lambda_{B_{1}, B_{2}, C}=0, \pm 1$ (as opposed to $t$ ) only. We have given the association with the $\mathrm{SO}(3)$ Kronecker products and indicated the corresponding polarisation vectors in the subscripts. The dots stand for higher representations i.e. non-invariant objects which are of no concern to us.

Using the parametrization given in appendix A for the polarisation tensors and tables for CGC e.g., [10], the ratios of the HAs in both cases and both methods read ${ }^{2}$

$$
\begin{aligned}
& H_{t 0}: H_{t+}: H_{t-}=-1: 1: 1, \\
& H_{+-}: H_{+0}: H_{-0}=-1: 1: 1 .
\end{aligned}
$$

Other operators contributing to (2.1) can be analysed in analoguous manner with either method.

\subsection{Summary of endpoint relations for $B(D) \rightarrow V \ell \ell$}

Relations between HAs of operators $B(D) \rightarrow V \ell \ell$ are summarised in this section. Endpoint relations for $B(D) \rightarrow X_{J} \ell \ell$ for spin $J \neq 1$ are given in section 4 . The most generic dimension six operators are given by a total of 10 operators $\left(O_{\mathcal{T}}=1 / 2\left(O_{T}+O_{T_{5}}\right)\right.$ in the notation of $[8])$ :

$$
O_{S(P)}=\bar{s}_{L} b \bar{\ell}\left(\gamma_{5}\right) \ell, \quad O_{V(A)}=\bar{s}_{L} \gamma^{\mu} b \bar{\ell} \gamma_{\mu}\left(\gamma_{5}\right) \ell, \quad O_{\mathcal{T}}=\bar{s}_{L} \sigma^{\mu \nu} b \bar{\ell} \sigma_{\mu \nu} \ell, \quad O^{\prime}=\left.O\right|_{s_{L} \rightarrow s_{R}}
$$

\footnotetext{
${ }^{2}$ The results of the preprint v3 [8] are different in signs because of different conventions for the polarisation vectors (see footnote 3 for further remarks) and because of a mismatch of a factor $\sqrt{2}$ in $H_{t 0}$ and $H_{+-}$. The final expressions, by which we mean the differential decay rate, yields results which are consistent for the purpose of this work.
} 
We have assumed a $b \rightarrow s$ transition for the sake of explicitness. The corresponding Wilson coefficients carry the same sub and superscripts, e.g., $H_{\text {eff }} \propto C_{P}^{\prime} \bar{s}_{R} b \bar{\ell} \gamma_{5} \ell+\ldots$ Note, the notation $O_{V(A)} \propto O_{9(10)}$ is frequently used in the literature. The basis (2.14) involves scalars, vectors and tensors which correspond to no, one and two-antisymmetric Lorentz indices. Non-vanishing HAs can also arise when operators with two-symmetric Lorentz indices [11] are considered but the latter are of dimension eight and are therefore expected to be suppressed in generic models of new physics. The endpoint relation of $O_{\mathcal{T}}$ were analysed in the previous section. Armed with this knowledge and appendix A, the relations of the scalar and vector operators are easily obtained:

- scalars: at the endpoint the covariant objects are $\omega_{\mu}^{*}\left(\lambda_{C}\right)$ and $\omega_{\mu}^{*}(t)$, which can be combined into a scalar product which vanishes. Hence scalars do not contribute at the endpoint.

- vectors: the covariant objects are $\omega_{\mu}^{*}\left(\lambda_{C}\right), \omega_{\mu}^{*}(t)$ and $\omega_{\mu}^{*}\left(\bar{\lambda}_{B_{1}}\right)$. The only non-vanishing invariant at the endpoint is $\omega_{\mu}^{*}\left(\lambda_{C}\right) \omega^{* \mu}\left(\bar{\lambda}_{B_{1}}\right)=-(-1)^{\lambda_{C}} \delta_{\lambda_{C} \lambda_{B_{1}}}$, see (A.3).

We summarise the endpoint relations, ${ }^{3}$ valid at $q^{2}=q_{\max }^{2}=\left(m_{B}-m_{V}\right)^{2}$, as follows:

$$
\begin{array}{lll}
\text { scalars }\left(O_{S, P}^{\left({ }^{\prime}\right)}\right): & H=0, & \\
\text { vectors }\left(O_{V, A}^{\left({ }^{\prime}\right)}\right): & H_{0}=-H_{+}=-H_{-}, & \\
& H_{t}=0, \\
\text { tensors }\left(O_{\mathcal{T}}^{\left({ }^{\prime}\right)}\right): & H_{+-}=-H_{+0}=-H_{-0}, & {\left[H_{\|}^{\mathcal{T}}=-\sqrt{2} H_{0}, H_{\perp}^{\mathcal{T}}, H_{\perp}^{\mathcal{T}}=0\right],} \\
& H_{t 0}=-H_{t+}=-H_{t-} \quad\left[H_{\|}^{\mathcal{T}_{t}}=-\sqrt{2} H_{0}^{\mathcal{T}_{t}}, H_{\perp}^{\mathcal{T}_{t}}=0\right],
\end{array}
$$

where we have given the equivalent relations in the transversity basis, defined in the next section, in square brackets. The relevance of $O$ versus $O^{\prime}$ in terms of selection rules of powers of the three momentum $\kappa$ is discussed in the next section as well. The polarisation of the leptons, e.g., $O_{S(V)}$ vs $O_{P(A)}$ is immaterial as the relations (2.15) describe the $B \rightarrow V$ transition and do not specify the leptons in any way. Considering them can though provide additional information. For instance, $H_{t}$ vanishes or is proportional to a $m_{\ell} O_{P}$-contribution when $H_{t}$ is contracted with a vector or axial lepton bilinear, respectively, after using the equations of motion. One might therefore write $m_{\ell} H_{t} \rightarrow H_{t}^{\text {eff }}$ and absorb it into $O_{P}$ and vice versa. We note that $H_{t}, H=0$ is consistent with the latter statement.

\subsection{Parity selection rules the transversity amplitudes}

At the endpoint there are stringent selection rules on the HAs [3]. Following the same line of arguments we extend some of those results to include timelike polarisation and obtain

$$
\mathrm{HA} \propto C_{+} \kappa^{n}+C_{-} \kappa^{n \pm 1}, \quad C_{ \pm} \equiv C \pm C^{\prime},
$$

\footnotetext{
${ }^{3}$ Note that polarisation vectors are frequently chosen such that $H_{0}=H_{1}=H_{\overline{1}}$ and therefore $H_{\|}=$ $\sqrt{2} H_{0}$. We shall not do so as we stick to the Jacob-Wick and Condon-Shortley conventions of the second helicity state and CGC. Of course these conventions drop out in physical observables.
} 


\begin{tabular}{|l||c||c|c|c||c|c|c|c|}
\hline $\mathrm{HA}$ & $H$ & $H_{t}$ & $H_{0, \|}$ & $H_{\perp}$ & $H_{0, \|}^{\mathcal{T}_{t}}$ & $H_{\perp}^{\mathcal{T}_{t}}$ & $H_{0, \|}^{\mathcal{T}}$ & $H_{\perp}^{\mathcal{T}}$ \\
\hline WCC & $C_{-}$ & $C_{-}$ & $C_{-}$ & $C_{+}$ & $C_{-}$ & $C_{+}$ & $C_{+}$ & $C_{-}$ \\
$\propto \kappa^{n}$ & $\kappa+\ldots$ & $\kappa+\ldots$ & $\kappa^{0}+\ldots$ & $\kappa+\ldots$ & $\kappa^{0}+\ldots$ & $\kappa+\ldots$ & $\kappa^{0}+\ldots$ & $\kappa+\ldots$ \\
\hline
\end{tabular}

Table 1. Dependence of the HAs on the Wilson coefficient combination (WCC) for $B\left(0^{-}\right) \rightarrow$ $V\left(1^{-}\right) \ell \ell$ decays. The combination $C_{ \pm} \equiv C \pm C^{\prime}$ corresponds to a parity conserving (parity violating) coupling for the $b \rightarrow s$ transition. Together with the rule (2.16) and the discussion in the text this implies the power-behaviour in the three-momentum $\kappa$ as indicated in the last row. For particles with opposite internal parities $\eta=-1$ but identical spin assignment, the substitutions $C_{ \pm} \rightarrow C_{\mp}$ are the only requisite change to this table. An example is given by the decay $B\left(0^{-}\right) \rightarrow K_{1}(1270)\left(1^{+}\right) \ell$.

where $\kappa$ is the absolute value of the three momentum of the $C$-particle in the A restframe. It is proportional to the Källén-function $\lambda$ :

$\kappa=\left|\overrightarrow{q_{B}}\right|=\left|\overrightarrow{q_{C}}\right|=\sqrt{\frac{\lambda\left(q_{A}^{2}, q_{B}^{2}, q_{C}^{2}\right)}{4 q_{A}^{2}}}, \quad \lambda\left(q_{A}^{2}, q_{B}^{2}, q_{C}^{2}\right) \equiv\left(q_{A}^{2}-\left(q_{B}+q_{C}\right)^{2}\right)\left(q_{A}^{2}-\left(q_{B}-q_{C}\right)^{2}\right)$,

where $q_{r} \equiv \sqrt{q_{r}^{2}}$. The Wilson coefficients $C$ and $C^{\prime}$ correspond to left handed and right handed $s$ quarks in the transition operator, respectively (2.14).

In general if parity is conserved then the S-matrix and therefore the amplitude is an even(odd) power in the external momenta if the product of the internal parities of the initial and final state particles are 1(-1) [12]. It is therefore advantageous to consider HAs of definite parity (transversity basis):

$$
\begin{aligned}
& H_{\|(\perp)} \equiv \frac{1}{\sqrt{2}}\left(H_{+} \pm H_{-}\right), \\
& H_{\|(\perp)}^{\mathcal{T}_{t}} \equiv \frac{1}{\sqrt{2}}\left(H_{t+} \pm H_{t-}\right), \quad H_{0}^{\mathcal{T}_{t}} \equiv H_{t 0} \\
& H_{\|(\perp)}^{\mathcal{T}} \equiv \frac{1}{\sqrt{2}}\left(H_{+0} \pm H_{-0}\right), \quad H_{0}^{\mathcal{T}} \equiv H_{+-} .
\end{aligned}
$$

To assess a definite parity, consider the HA for the $B\left(0^{-}\right) \rightarrow V\left(1^{-}\right)$transition, which can be written as

$$
H_{\lambda_{B_{1}} \ldots \lambda_{B_{X}}} \propto\left\langle V\left|\bar{b} \Gamma_{\mu_{1} \ldots \mu_{X}} s\right| B\right\rangle \beta^{\mu_{1}}\left(\lambda_{B_{1}}\right) \ldots \beta^{\mu_{X}}\left(\lambda_{B_{X}}\right)
$$

The internal parity is $\eta=\eta_{B\left(0^{-}\right)} \eta_{V\left(1^{-}\right)}=(-1)(-1)=1$. The parity of the transition is given by the parity of the Dirac bilinear contracted with the polarisation vectors. Concerning the latter one needs to take into consideration that $\beta_{\mu}(t)$ and $\beta_{\mu}(i),(i=0, \pm)$ transform as vectors $J^{P}=1^{-}$and pseudo-vectors $J^{P}=1^{+}$respectively. Consequences of parity covariance are summarised for $B\left(0^{-}\right) \rightarrow V\left(1^{-}\right) \ell$ in table 1 . The dots stand for corrections which are of relative order $\mathcal{O}\left(\kappa^{2}\right)$ by virtue of parity and analyticity.

We note that exchanging a spacelike with a timelike polarisation index selects the opposite chirality combination of Wilson coefficients in agreement with the transformation properties mentioned earlier. Table 1 is consistent with the literature e.g. [8]. We would 
like to add that it equally applies for radially excited mesons. The rules are easily adapted to different internal parities as described in the caption of the table.

\section{Semileptonic $B$ and $D$ decays into vector mesons}

The endpoint relations (2.15) are of direct relevance for observables as the differential decay rate is proportional to two powers of the HAs. Below we continue to use $\bar{B} \rightarrow \bar{K}^{*}(\rightarrow$ $\bar{K} \pi) \ell^{+} \ell^{-}$as a template for $S \rightarrow V \ell \nu, S \rightarrow V \ell^{+} \ell^{-}$and $S \rightarrow V \nu \bar{\nu}$, where $S=B_{(s, c)}, D_{(s)}$, $\ell=e, \mu, \tau$, and $V$ denotes a vector meson generally observed through the decay into two pseudoscalar ones, see section 3.4. Applications to non-vector modes are discussed in section 4 .

The starting point for phenomenological implications is the $\bar{B} \rightarrow \bar{K}^{*}(\rightarrow \bar{K} \pi) \ell^{+} \ell^{-}$ angular distribution $[13]^{4}$ for the operator basis (2.14):

$$
\begin{aligned}
\frac{8 \pi}{3 \kappa \kappa_{\ell}} \frac{d^{4} \Gamma}{d q^{2} d \cos \theta_{\ell} d \cos \theta_{K} d \phi}= & \left(J_{1 s}+J_{2 s} \cos 2 \theta_{\ell}+J_{6 s} \cos \theta_{\ell}\right) \sin ^{2} \theta_{K} \\
& +\left(J_{1 c}+J_{2 c} \cos 2 \theta_{\ell}+J_{6 c} \cos \theta_{\ell}\right) \cos ^{2} \theta_{K} \\
& +\left(J_{3} \cos 2 \phi+J_{9} \sin 2 \phi\right) \sin ^{2} \theta_{K} \sin ^{2} \theta_{\ell} \\
& +\left(J_{4} \cos \phi+J_{8} \sin \phi\right) \sin 2 \theta_{K} \sin 2 \theta_{\ell} \\
& +\left(J_{5} \cos \phi+J_{7} \sin \phi\right) \sin 2 \theta_{K} \sin \theta_{\ell},
\end{aligned}
$$

where $\theta_{\ell}$ denotes the angle between the $\ell^{-}$and $\bar{B}$ in the $\left(\ell^{+} \ell^{-}\right)$center of mass system $(\mathrm{cms})$, $\theta_{K}$ the angle between $K^{-}$and $\bar{B}$ in the $\left(K^{-} \pi^{+}\right)$cms and $\phi$ the angle between the two decay planes spanned by the 3 -momenta of the $\left(K^{-} \pi^{+}\right)$- and $\left(\ell^{+} \ell^{-}\right)$-systems, respectively. The variable $q^{2}$ denotes the invariant mass-squared of the dileptons. The angular coefficients $J_{i}=J_{i}\left(q^{2}\right)$ expressed in terms of HAs in the most general dimension six operator basis including finite lepton masses is given in ref. [8]. The phase space factor $\kappa \kappa_{\ell}$ is defined in eq. (C.1).

From table 1 we infer that there are four non-vanishing HAs, $H^{V, A}$ and $H^{\mathcal{T}, \mathcal{T}_{t}}$ of the vector and tensor type. Since there are a total of twelve angular functions $J_{i x}$, we therefore expect at least 8 relations among them. In fact we find that there are ten relations:

$$
\begin{array}{ll}
J_{2 s}\left(q_{\max }^{2}\right)=-J_{2 c}\left(q_{\max }^{2}\right) / 2, & J_{1 s}\left(q_{\max }^{2}\right) \quad-J_{2 s}\left(q_{\max }^{2}\right) / 3=J_{1 c}\left(q_{\max }^{2}\right)-J_{2 c}\left(q_{\max }^{2}\right) / 3, \\
J_{3}\left(q_{\max }^{2}\right)=-J_{4}\left(q_{\max }^{2}\right), & J_{2 c}\left(q_{\max }^{2}\right)=J_{3}\left(q_{\max }^{2}\right), \quad J_{5,6 s, 6 c, 7,8,9}\left(q_{\max }^{2}\right)=0,
\end{array}
$$

which is the same number as for vector operators only. Above $q_{\max }^{2}=\left(m_{B}-m_{K^{*}}\right)^{2}$ denotes the kinematic endpoint in the $q^{2}$-variable. Many of them follow from $H_{\perp}\left(q_{\max }^{2}\right)=$ $H_{\perp}^{\mathcal{T}}\left(q_{\max }^{2}\right)=H_{\perp}^{\mathcal{T}_{t}}\left(q_{\max }^{2}\right)=0$ which in turn is a consequence of parity covariance alone. We stress that the relations (3.2) are independent of the dynamics. For the CP-conjugated mode $B \rightarrow K^{*}(\rightarrow K \pi) \ell^{+} \ell^{-}$the $J_{i}$ transform as $\bar{J}_{1,2,3,4,7}=J_{1,2,3,4,7}$ and $\bar{J}_{5,6,8,9}=-J_{5,6,8,9}$ with all weak phases conjugated in addition. This is due to the convention that the angle $\theta_{\ell}$ is defined with respect to the same negatively charged lepton in both $B$ and $\bar{B}$ decays which implies the transformations $\theta_{l} \rightarrow \theta_{l}-\pi$ and $\phi \rightarrow-\phi$ upon CP-conjugation.

\footnotetext{
${ }^{4}$ We have taken out the overall phase space factor $\kappa \kappa_{\ell}$, which drops out in ratios. That is to say the decay rate is schematically given as $d \Gamma \propto \kappa \kappa_{\ell}|\mathrm{HA}|^{2} d$ (phase space).
} 


\subsection{Kinematic endpoint: observables in a general dimension six operator basis}

The relations (3.2) imply that many observables considered obey exact relations at the kinematic endpoint. They give rise to isotropic uniangular distributions ${ }^{5}$

$$
\begin{array}{ll}
\frac{d^{2} \Gamma}{d \cos \theta_{\ell} d q^{2}} /\left(\frac{d \Gamma}{d q^{2}}\right)= & \rightarrow \frac{1}{2}, \\
\kappa \kappa_{\ell}\left(\left(J_{1 s}+\frac{J_{1 c}}{2}\right)+\left(J_{6 s}+\frac{J_{6 c}}{2}\right) \cos \theta_{\ell}+\left(J_{2 s}+\frac{J_{2 c}}{2}\right) \cos 2 \theta_{\ell}\right) /\left(\frac{d \Gamma}{d q^{2}}\right) & \rightarrow \frac{1}{2}, \\
\frac{d^{2} \Gamma}{d \cos \theta_{K} d q^{2}} /\left(\frac{d \Gamma}{d q^{2}}\right)=\kappa \kappa_{\ell} \frac{3}{2}\left(\left(J_{1 s}-\frac{J_{2 s}}{3}\right) \sin ^{2} \theta_{K}+\left(J_{1 c}-\frac{J_{2 c}}{3}\right) \cos ^{2} \theta_{K}\right) /\left(\frac{d \Gamma}{d q^{2}}\right) &
\end{array}
$$

where $d \Gamma / d q^{2}=3 \kappa \kappa_{\ell}\left(J_{1 s}-1 / 3 J_{2 s}\right)$. The interpretation of (3.3) is that at the endpoint the $\ell \ell$ - and $K \pi$-pair are in the $l=0$ (S-wave) spherically symmetric partial wave configuration. Eq.(3.3) is also consistent with:

$$
\begin{aligned}
& F_{L}\left(q_{\max }^{2}\right)=\kappa \kappa_{\ell}\left(J_{1 c}-\frac{1}{3} J_{2 c}\right) /\left(\frac{d \Gamma}{d q^{2}}\right)=\frac{1}{3}, \\
& A_{\mathrm{FB}}\left(q_{\max }^{2}\right)=\kappa \kappa_{\ell}\left(J_{6 s}+\frac{J_{6 c}}{2}\right) /\left(\frac{d \Gamma}{d q^{2}}\right)=0 .
\end{aligned}
$$

The uniangular distribution in the angle $\phi$ is given by

$$
\frac{d^{2} \Gamma}{d \phi d q^{2}} /\left(\frac{d \Gamma}{d q^{2}}\right)=\frac{1}{2 \pi}\left(1+r_{\phi} \cos 2 \phi\right), \quad r_{\phi} \equiv \frac{-8 J_{2 s}}{9\left(J_{1 s}-1 / 3 J_{2 s}\right)}
$$

which is not isotropic in general. This is to be expected since $\phi$ is the angle between the two decay planes which has no special rôle at the kinematic endpoint. The result for $r_{\phi}$ in the general dimension six operator basis is given in appendix E. In the SM + SM' operator basis $\left(O_{V(A)}\right.$ and primed (2.14) only) one obtains in this basis $r_{\phi}=-1 / 3+\mathcal{O}\left(m_{\ell}^{2} / m_{b}^{2}\right)$. This can be tested experimentally and deviations thereof could be explained by the tensor operator contributions as given in appendix E.

The vanishing of $J_{5,6 s, 6 c, 7,8,9}\left(q_{\max }^{2}\right)$ implies, besides $A_{\mathrm{FB}}\left(q_{\max }^{2}\right)=0$, for the CPasymmetries $A_{i}^{(D)} \propto J_{i}-\bar{J}_{i}[14]$ and symmetries $\left.S_{i} \propto J_{i}+\bar{J}_{i}[15]\right)$,

$$
A_{5,6,7,8,9}^{(D)}\left(q_{\max }^{2}\right)=0, \quad S_{5,6,7,8,9}\left(q_{\max }^{2}\right)=0
$$

and for the related ones $P_{k}^{\prime}[16]$

$$
P_{5,6,8}^{\prime}\left(q_{\max }^{2}\right)=0, \quad P_{4}^{\prime}\left(q_{\max }^{2}\right)=\sqrt{2}
$$

\footnotetext{
${ }^{5}$ The angles are defined at the endpoint through a limiting procedure only.
} 
For the transverse asymmetry $A_{T}^{(2)}=J_{3} /\left(2 J_{2 s}\right)[17] A_{T}^{(2)}\left(q_{\max }^{2}\right)=-1$ holds. The low recoil observables $H_{T}^{(i)}[7]$

$$
\begin{aligned}
H_{T}^{(1)} \equiv \frac{\sqrt{2} J_{4}}{\sqrt{-J_{2 c}\left(2 J_{2 s}-J_{3}\right)}}, & H_{T}^{(1 b)} \equiv \frac{J_{2 c} J_{6 s}}{2 J_{4} J_{5}}, & H_{T}^{(2)} \equiv \frac{\beta_{\ell} J_{5}}{\sqrt{-2 J_{2 c}\left(2 J_{2 s}+J_{3}\right)}}, \\
H_{T}^{(3)} \equiv \frac{\beta_{\ell} J_{6 s}}{2 \sqrt{\left(2 J_{2 s}\right)^{2}-J_{3}^{2}}}, & H_{T}^{(4)} \equiv \frac{2 J_{8}}{\sqrt{-2 J_{2 c}\left(2 J_{2 s}+J_{3}\right)}}, & H_{T}^{(5)} \equiv-\frac{2 J_{9}}{2 \sqrt{\left(2 J_{2 s}\right)^{2}-J_{3}^{2}}},
\end{aligned}
$$

with $\beta_{\ell}$ defined in eq. (C.1), obey

$$
\left|H_{T}^{(1)}\left(q_{\max }^{2}\right)\right|=1, \quad H_{T}^{(1 b)}\left(q_{\max }^{2}\right)=1, \quad \frac{H_{T}^{(2)}\left(q_{\max }^{2}\right)}{H_{T}^{(3)}\left(q_{\max }^{2}\right)}=1, \quad \frac{H_{T}^{(4)}\left(q_{\max }^{2}\right)}{H_{T}^{(5)}\left(q_{\max }^{2}\right)}=1 .
$$

The sign of $H_{T}^{(1)}$ depends on the sign of the 0-helicity amplitude. Note that in the second, third and fourth relation both the nominators and the denominators are linear in $\kappa$ (c.f section 3.2).

Such relations have various applications. For instance they impose constraints on parameterizations of form factors and fits to decay distributions, see e.g. recently [9] or they serve as cross checks for experimental analyses. We checked explicitly that the relations eqs. (3.2)-(3.9) are obeyed by the low recoil OPE results in the most general dimension six operator basis [8].

Note that an experimental extraction of the angular coefficients in (3.1) and derived observables requires to some extent non-local (in $q^{2}$ ) information, such as from binning or fit shapes. While the exact size of the corresponding corrections will depend on the observable at hand, see ref. [18] for such a study and section 3.3.1 for comments, one expects them to be controlled for a sufficiently small bin at endpoint. Near the endpoint the variation in $q^{2}$, due to possible resonances (see section 3.3 for related discussions), is relatively mild. We conclude that endpoint relations such as (3.3)-(3.5) are asymptotically observable.

\subsection{Small momentum expansion (low recoil): the SM + SM' basis}

We consider the observables of the previous section in the vicinity of the endpoint within the SM + SM' basis. In addition we employ the approximation $m_{\ell}=0$. This means that we only consider (axial-) vector operators $O_{V(A)}$ and the primed ones, which result in $H_{0, \|, \perp}^{x}$ $(x=L, R)$ amplitudes. Using the results in table 1 we parameterise the transversity basis near the endpoint as follows:

$$
H_{\|}^{x}=-\sqrt{2} H_{0}^{x}=a_{\|}^{x}+\mathcal{O}\left(\kappa^{2}\right), \quad H_{\perp}^{L, R}=a_{\perp}^{x} \kappa+\mathcal{O}\left(\kappa^{3}\right), \quad x=L, R,
$$

where $\kappa$ is the absolute value of the three momentum of the $K^{*}$-meson in the $B$ restframe. The relation between the three momentum $\kappa$ and $q^{2}$ is given in (2.17). More details as well as phenomenological parameterisations for low and high $q^{2}$ including further terms in the $\kappa$-expansion are briefly sketched in appendix C. 
In this work we neglect $\mathrm{CP}$-violation, because in $B \rightarrow K^{*}$ transitions direct $\mathrm{CP}$ violation is small in the $\mathrm{SM}$ and there is no experimental evidence for it presently. We remark that CP-violating effects to the parameterisations could be included by simply introducing the analogous parameters for $\mathrm{CP}$-conjugated quantities, allowing to form $\mathrm{CP}$ averaged observables and CP-asymmetries. Expanding up to linear order in $\kappa$ one obtains:

$$
\begin{aligned}
& J_{1 s}=\frac{3}{4}|A|^{2}, \quad J_{1 c}=\frac{1}{2}|A|^{2}, \quad J_{2 s}=\frac{1}{4}|A|^{2}, \quad J_{2 c}=-\frac{1}{2}|A|^{2}, \quad J_{3}=-\frac{1}{2}|A|^{2}, \quad J_{4}=\frac{1}{2}|A|^{2}, \\
& J_{5}=R \kappa, \quad J_{6 c}=0, \quad J_{6 s}=2 R \kappa, \quad J_{7}=0, \quad J_{8}=\frac{1}{2} I \kappa, \quad J_{9}=-I \kappa,
\end{aligned}
$$

with further corrections of relative order $\mathcal{O}\left(\kappa^{2}\right)$. Here we use the following:

$$
\begin{aligned}
|A|^{2} & \equiv\left|a_{\|}^{L}\right|^{2}+\left|a_{\|}^{R}\right|^{2}=\frac{1}{2}\left(\left|a_{\|}^{V}\right|^{2}+\left|a_{\|}^{A}\right|^{2}\right), \\
R & \equiv \operatorname{Re}\left[a_{\|}^{L} a_{\perp}^{L *}-a_{\|}^{R} a_{\perp}^{R *}\right]=-\frac{1}{2} \operatorname{Re}\left[a_{\|}^{V} a_{\perp}^{A *}+a_{\|}^{A} a_{\perp}^{V *}\right], \\
I & \equiv \operatorname{Im}\left[a_{\|}^{L} a_{\perp}^{L *}+a_{\|}^{R} a_{\perp}^{R *}\right]=\frac{1}{2} \operatorname{Im}\left[a_{\|}^{V} a_{\perp}^{V *}+a_{\|}^{A} a_{\perp}^{A *}\right],
\end{aligned}
$$

and $a^{V / A}=a^{R} \pm a^{L}$. The relations (3.11) are compatible with (3.2) in the limit $\kappa \rightarrow 0$ and match the expressions in chapter III.B of ref. [8] to the given order. We stress that (3.11) is though independent of any dynamical assumptions. Observables linear in $\kappa$ are:

$$
\begin{aligned}
A_{\mathrm{FB}} & =\frac{J_{6 s}-J_{6 c} / 2}{3 J_{1 s}-J_{2 s}}=\hat{R} \kappa, \quad P_{5}^{\prime}=\frac{J_{5}}{2 \sqrt{-J_{2 c} J_{2 s}}}=\sqrt{2} \hat{R} \kappa, \\
S_{8} & =\frac{4 / 3 J_{8}}{3 J_{1 s}-J_{2 s}}=\frac{1}{3} \hat{I} \kappa, \quad S_{9}=\frac{4 / 3 J_{9}}{3 J_{1 s}-J_{2 s}}=-\frac{2}{3} \hat{I} \kappa,
\end{aligned}
$$

where

$$
\hat{R} \equiv \frac{R}{|A|^{2}}, \quad \hat{I} \equiv \frac{I}{|A|^{2}} .
$$

Consequently, in the vicinity of the endpoint the following relations hold,

$$
\frac{P_{5}^{\prime}}{A_{\mathrm{FB}}}=\sqrt{2}+\mathcal{O}\left(\kappa^{2}\right), \quad \frac{S_{8}}{S_{9}}=-\frac{1}{2}+\mathcal{O}\left(\kappa^{2}\right), \quad \frac{H_{T}^{(4)}}{H_{T}^{(2)}}=\frac{\hat{I}}{\hat{R}}+\mathcal{O}\left(\kappa^{2}\right) .
$$

We compare the endpoint predictions with existing data from BaBar [19], CDF [20], LHCb [21-23], ATLAS [24] and CMS [25] in table 2. The data are consistent with the endpoint relations. The largest deviation is at the $2 \sigma$-level in the statistics-limited $B_{s} \rightarrow$ $\phi \mu^{+} \mu^{-}$analysis [22]. The prediction for the ratio $P_{5}^{\prime} / A_{\mathrm{FB}}$ is satisfied at $1 \sigma$. Since both measurements of $S_{8}$ and $S_{9}$ are currently consistent with zero, the corresponding ratio comes with a large uncertainty. The perfect agreement of the present experimental central value with the endpoint prediction has therefore to be seen as accidental.

Investigating the observables linear in $\kappa$ we find from table 2

$$
\hat{R}=(-0.67 \pm 0.07) \mathrm{GeV}^{-1}, \quad \hat{I}=(-0.17 \pm 0.27) \mathrm{GeV}^{-1}, \quad \hat{I} / \hat{R}=0.25 \pm 0.40 .
$$




\begin{tabular}{|c|c|c|c|c|c|c|c|c|c|c|}
\hline & $F_{L}$ & $S_{3}$ & ${ }^{a} P_{4}^{\prime}$ & $S_{7}$ & ${ }^{b} P_{5}^{\prime} / A_{\mathrm{FB}}$ & ${ }^{b} S_{8} / S_{9}$ & ${ }^{a} A_{\mathrm{FB}}$ & $P_{5}^{\prime}$ & ${ }^{a} S_{8}$ & $S_{9}$ \\
\hline endpoint & $1 / 3$ & $-1 / 3$ & $\sqrt{2}$ & 0 & $\sqrt{2}$ & $-1 / 2$ & $\hat{R} \kappa$ & $\sqrt{2} \hat{R} \kappa$ & $1 / 3 \hat{I} \kappa$ & $-2 / 3 \hat{I} \kappa$ \\
\hline$B \rightarrow K^{*}$ & $0.38 \pm 0.04$ & $-0.22 \pm 0.09$ & $0.70_{-0.52}^{+0.44}$ & $0.15_{-0.15}^{+0.16}$ & $1.63 \pm 0.57$ & $-0.5 \pm 2.2$ & $-0.36 \pm 0.04$ & $-0.60_{-0.18}^{+0.21}$ & $-0.03 \pm 0.12$ & $0.06_{-0.10}^{+0.11}$ \\
\hline$B_{s} \rightarrow \phi$ & $0.16_{-0.12}^{+0.18}$ & $0.19_{-0.31}^{+0.30}$ & - & - & - & - & - & - & - & - \\
\hline
\end{tabular}

Table 2. Comparison of endpoint predictions (second row) for angular observables (first row) to the current world average in the available endpoint-bin $q^{2} \in[16,19] \mathrm{GeV}^{2}$ (LHC-experiments) or otherwise $q^{2} \in\left[16 \mathrm{GeV}^{2}, q_{\max }^{2}\right]$ for $B \rightarrow K^{*} \ell^{+} \ell^{-}$decays (third row; our error weighted average, systematic and statistical uncertainties are added in quadrature). The last row gives corresponding data for $B_{s} \rightarrow \phi \mu^{+} \mu^{-}$decays [22] (LHCb only). ${ }^{a}$ Experimental values adopted to theory definitions as in [8] $\left(A_{\mathrm{FB}}\right),[26]\left(S_{i}\right)$ and [16] $\left(P_{k}^{\prime}\right) .{ }^{b}$ with symmetrized errors and assuming gaussian error propagation. Note, $S_{3}=1 / 2\left(1-F_{L}\right) A_{T}^{(2)}$. All corrections are of relative order $\mathcal{O}\left(\kappa^{2}\right)\left(\right.$ for $\left.m_{\ell}=0\right)$ by virtue of parity covariance.

The bin-averaged 3-momenta are $\kappa_{\text {bin }}=0.55 \mathrm{GeV}$ for $q^{2} \in[16,19] \mathrm{GeV}^{2}$ and $\kappa_{\text {bin }}=$ $0.52 \mathrm{GeV}$ for $q^{2} \in\left[16 \mathrm{GeV}^{2}, q_{\text {max }}^{2}\right]$. The measured value of $\hat{R}$ is in accordance with its SM prediction, $\hat{R}_{\mathrm{SM}}=\left(-0.73_{-0.13}^{+0.12}\right) \mathrm{GeV}^{-1}[8]$. In the limit where the strong and the weak phases do not depend on the polarisation, or both phases are negligible, $\hat{I}=0$ holds. In the SM $\hat{I}=0$ (which is consistent with [7]) since there are no sizeable weak phase differences and as argued in the next section the leading strong phases are polarisation independent indeed. The current experimental results in (3.16) are therefore consistent with the SM.

\subsection{The validity of small momentum expansion and $c \bar{c}$-resonances}

To evaluate the performance of the $\kappa$-expansion we discuss in section 3.3.1 the (non)-impact of charmonium resonances in the low recoil region, and compare suitable observables at low recoil to data (section 3.3.2). In section 3.3.3 we discuss possible experimental probes of non-factorizable contributions in this region.

\subsubsection{The (non-)impact of the $c c$-resonances at low recoil}

Charm-resonances [10] contribute to $B \rightarrow K^{(*)} \ell \ell$ via $B \rightarrow K^{(*)}\left(c \bar{c} \rightarrow \gamma^{*} \rightarrow \ell \ell\right)$ and are visible in the local spectrum starting from $q^{2}=m_{J / \Psi}^{2} \simeq 9.6 \mathrm{GeV}^{2}$ onwards. While such effects have been known to the theory community e.g., [27-29], the pronounced structure observed recently in the low recoil region in the $B \rightarrow K \mu^{+} \mu^{-}$dimuon spectrum [30] reinforces one to think about the most suitable binnings. Here we would like to point out that for observables composed out of certain ratios of HAs the situation is simpler since factorizable (universal) contributions, which are supposedly leading, drop out as they are polarisation independent. To show this we write the HAs, neglecting right handed currents, as follows:

$$
\begin{array}{ll}
H_{i}^{V}=F_{i}^{V}\left(q^{2}\right)\left(1+L^{\mathrm{fac}, \mathrm{c}}\left(q^{2}\right)+L_{i}^{\mathrm{n}-\mathrm{fac}, \mathrm{c}}\left(q^{2}\right)+\ldots\right), & \\
H_{i}^{A}=F_{i}^{A}\left(q^{2}\right)(1+\ldots), & i=\perp, \|, 0,
\end{array}
$$

where $F_{i}^{V(A)}\left(q^{2}\right) \propto C_{V(A)} f_{i}\left(q^{2}\right), L^{\text {fac,c }}$ and $L_{i}^{\text {n-fac,c }}$ denote the non-resonant (e.g. $F_{\|}^{V(A)} \propto$ $C_{9(10)} A_{1}\left(q^{2}\right)$ in the conventions of [8]), factorizable charm and non-factorizable charm contribution, respectively. The ellipses stand for all other terms. The charm loop with no 
gluon reconnecting to any other part of the diagram is part of $L^{\mathrm{fac}, \mathrm{c}}$ (naive factorization) and the charm loop with gluons emitted into the vector meson final state constitutes a part of $L_{\lambda}^{\mathrm{n} \text {-fac,c }}$. Similar to the cancellation of (universal) short-distance coefficients, universal charm-contributions $L^{\text {fac,c }}$ drop out in observables which are ratios of certain combinations of HAs composed of the sum of squares as $\left(H_{i}^{L} H_{j}^{L *}+H_{i}^{R} H_{j}^{R *}\right) /\left(H_{l}^{L} H_{k}^{L *}+H_{l}^{R} H_{k}^{R *}\right)$, where $i, j, k, l=\perp, \|, 0[7]$. This can also be seen by defining

$$
\begin{aligned}
x_{i j}(a) & \equiv\left(H_{i}^{V} H_{j}^{V *}+a H_{i}^{A} H_{j}^{A *}\right) \\
& =(1+a)\left(H_{i}^{L} H_{j}^{L *}+H_{i}^{R} H_{j}^{R *}\right)+(1-a)\left(H_{i}^{L} H_{j}^{R *}+H_{i}^{R} H_{j}^{L *}\right) \\
& \propto f_{i}\left(q^{2}\right) f_{j}^{*}\left(q^{2}\right)\left(\left|C_{V}\right|^{2}\left|1+L^{\mathrm{fac}, \mathrm{c}}\left(q^{2}\right)\right|^{2}+a\left|C_{A}\right|^{2}\right)+\mathcal{O}\left(L^{\mathrm{n}-\mathrm{fac}, \mathrm{c}}\right),
\end{aligned}
$$

where $a$ is a complex number. Thus for observables of the form,

$$
\begin{aligned}
\Phi\left(x_{i j}(a), \ldots, x_{l k}(a)\right), \quad \Phi(b x, \ldots, b y)= & \Phi(x, \ldots, y) \\
& \text { with } b \text { a number and } i, j, k, l=\perp, \|, 0,
\end{aligned}
$$

the $L^{\text {fac,c }}$-contribution effectively drops out. Examples of observables of the form (3.19) are:

$$
\begin{aligned}
F_{L} & \equiv \frac{\left|H_{0}^{L}\right|^{2}+\left|H_{0}^{R}\right|^{2}}{\sum_{X=L, R}\left(\left|H_{0}^{X}\right|^{2}+\left|H_{\perp}^{X}\right|^{2}+\left|H_{\|}^{X}\right|^{2}\right)}, \\
A_{T}^{(2)} & \equiv \frac{\left|H_{\perp}^{L}\right|^{2}+\left|H_{\perp}^{R}\right|^{2}-\left(\left|H_{\|}^{L}\right|^{2}+\left|H_{\|}^{R}\right|^{2}\right)}{\left|H_{\perp}^{L}\right|^{2}+\left|H_{\perp}^{R}\right|^{2}+\left|H_{\|}^{L}\right|^{2}+\left|H_{\|}^{R}\right|^{2}}, \\
P_{4}^{\prime} & \equiv \frac{\sqrt{2} \operatorname{Re}\left(H_{0}^{L} H_{\|}^{L *}+H_{0}^{R} H_{\|}^{R *}\right)}{\sqrt{\left(\left|H_{\perp}^{L}\right|^{2}+\left|H_{\perp}^{R}\right|^{2}+\left|H_{\|}^{L}\right|^{2}+\left|H_{\|}^{R}\right|^{2}\right)\left(\left|H_{0}^{L}\right|^{2}+\left|H_{0}^{R}\right|^{2}\right)}},
\end{aligned}
$$

where the latter requires for $L^{\text {fac }}$ to cancel that there are no sizeable weak phase differences between the $H_{0}$ and $H_{\|}$HAs. Corresponding CP-asymmetries are given in [8,31]. We recall that the presence of right-handed currents $\left(C_{+} \neq C_{-}\right.$in table 1$)$ would affect the cancellations in observables like $A_{T}^{(2)}$. Whereas this poses no problem for the SM where $C_{+} \simeq C_{-}$it would make precise interpretations of beyond the SM right-handed currents in the low recoil region more difficult.

While the evaluation of non-factorizable contributions $L_{i}^{\mathrm{n}-\mathrm{fac}, \mathrm{c}}$, e.g. [35], remains an important task, we stress that the endpoint relations obtained in this work imply that these non-factorizable contributions become polarisation independent at the endpoints, see (B.3), i.e.,

$$
L_{\lambda}^{\mathrm{n} \text {-fac,c }}\left(q_{\max }^{2}\right)=L^{\mathrm{n}-\mathrm{fac}, \mathrm{c}}, \quad \lambda=0, \pm 1, \|, \perp
$$

\subsubsection{The small momentum expansion at work for $F_{L}$ and $A_{T}^{(2)}$}

The endpoint relations are subject to corrections away from the endpoint which we have parameterised in eq. (3.10) in terms of a $\kappa$-expansion. The uncertainty estimate $\mathcal{O}\left(\kappa^{2}\right)$ is in general hampered by the $c \bar{c}$-resonance effects. However, as pointed out in the previous section such effects essentially drop out in observables which are of the form (3.19). Hence the corrections to the HAs can be taken to be of the order $\mathcal{O}\left(\kappa^{2} / q^{2}\right)$ (where $\mathcal{O}\left(q^{2}\right)=\mathcal{O}\left(m_{B}^{2}\right)$ 


\begin{tabular}{|c|c|c|c|c|c|c|}
\hline$q^{2}\left[\mathrm{GeV}^{2}\right]$ & $\begin{array}{l}J / \Psi \\
9.59\end{array}$ & {$[10.09,12.86]$} & $\begin{array}{l}\Psi(2 S) \\
13.59\end{array}$ & {$[14.18,16]$} & {$[16, X]^{\dagger}$} & $\begin{array}{c}\chi_{c 1} \\
12.33\end{array}$ \\
\hline$F_{L}$ & $0.566 \pm 0.007$ & $0.48 \pm 0.05$ & $0.48 \pm 0.05$ & $0.38 \pm 0.05$ & $0.38 \pm 0.04$ & $0.77 \pm 0.08$ \\
\hline$A_{T}^{(2)_{*}}$ & $-0.008 \pm 0.025$ & $-0.36 \pm 0.30$ & $0.15 \pm 0.17$ & $0.07 \pm 0.25$ & $-0.64 \pm 0.27$ & $-0.74 \pm 0.35$ \\
\hline
\end{tabular}

Table 3. $F_{L}$ and $A_{T}^{(2)}$ measured in $B \rightarrow K^{*} \ell^{+} \ell^{-}$decays on [37, 38] and off-resonance [19-21, 24, 25] (our error weighted average, systematic and statistical uncertainties are added in quadrature). ${ }^{\dagger} X=19$ for LHC experiments and $X=q_{\max }^{2}$ otherwise. The last column corresponds to $B \rightarrow$ $K^{*}\left(\chi_{c 1} \rightarrow J / \Psi \gamma\right)$ decays [37] for which large non-factorizable corrections are expected. *Our evaluation of $J / \Psi, \Psi(2 S)$ and $\chi_{c 1}$ using eq. (3.21).

and $m_{K^{*}}$ cannot enter the denominator because the HAs have got to be smooth in the limit $\left.m_{K^{*}} \rightarrow 0[32]\right)$.

Neglecting the small $q^{2}$-dependence of $C_{9}^{\text {eff }}$ from quark loops as well as terms $\mathcal{O}\left(C_{7} / C_{9}^{\text {eff }}\right), H_{\|}\left(q^{2}\right) \propto A_{1}\left(q^{2}\right)$ and we can estimate the $q^{2}$-correction at the beginning of the last bin by looking at $R_{\text {bin }}=A_{1}\left(16 \mathrm{GeV}^{2}\right) / A_{1}\left(q_{\text {max }}^{2}\right)$. One obtains $R_{\text {bin }} \simeq 0.85$ for both the extrapolated LCSR results in [33] as well as the recent lattice results [34]. Thus $15 \%$ (which has to be compared with the naive parametric value $\kappa^{2} / q^{2}=0.06$ ) can be taken as a measure of the error at the end of the endpoint bin. The averaged error over the bin should be only half the size thus one might expect the parameterisation to hold within $10 \%$.

To further substantiate this we study $F_{L}(3.20)$ and $A_{T}^{(2)}$ (3.21), which belong to type (3.19). $F_{L}$ interpolates between $F_{L}(0)=0$ where the longitudinal mode decouples completely and $F_{L}\left(q_{\max }^{2}\right)=1 / 3(3.4)$ where all polarisations are equally probable. In the intermediated regime it assumes a maximum around $F_{L}=\mathcal{O}(0.8-0.9)$ as a result of the zero of the $A_{\mathrm{FB}}$ and the equivalence theorem cf. appendix D. $A_{T}^{(2)}$ interpolates between $A_{T}^{(2)}(0) \simeq 0$ due to $H_{+}^{V, A} \simeq 0$ by virtue of the $V$-A-interactions and $A_{T}^{(2)}\left(q_{\max }^{2}\right)=-1$.

The data on $F_{L}$ and $A_{T}^{(2)}$ for $B \rightarrow K^{*} \ell \ell$ is compiled in table 3 . The result $\left.F_{L}\right|_{\mathrm{LHCb}}=$ $0.523 \pm 0.005 \pm 0.010$ for $B_{s} \rightarrow J / \Psi \phi[36]$ shows as expected a similar pattern to $B \rightarrow J / \Psi K^{*}$ decays. The change of $F_{L}$ observed in the next-to endpoint bin $[14.18,16] \mathrm{GeV}^{2}$ is within the expected order ten percent range of $F_{L}=1 / 3$ and the sign of the change is consistent with the previously mentioned theoretical considerations. The changes in $A_{T}^{(2)}$ versus $q^{2}$ are larger and come presently with a large experimental uncertainty. A significant change is, however, expected as the observable is forced, as we argued, to change rather abruptly from $A_{T}^{(2)}\left(q_{\text {max }}^{2}\right)=-1$ to $\left.A_{T}^{(2)}\right|_{\text {low } q^{2}} \simeq 0$.

\subsubsection{Low recoil OPE versus non-factorizable corrections}

The absence of estimates of the non-factorizable contributions $L_{i}^{\text {n-fac,c }}\left(q^{2}\right)$ in (3.17) are a limiting factor for precision predictions in the low recoil region. Turning this argument around the sensitivity to such effects can be used to obtain experimental information on the size of the $L_{i}^{\mathrm{n} \text {-fac,c }}\left(q^{2}\right)$. In this context $F_{L}$ and $A_{T}^{(2)}$ are useful as factorizable $c \bar{c}$-effects effectively drop out. In figure 2 we show $F_{L}$ and $A_{T}^{(2)}$ from $B \rightarrow K^{*} \ell^{+} \ell^{-}$decay data on (blue points) and off $c \bar{c}$-resonance (blue boxes) as in table 3 . For the predictions in the SM basis 

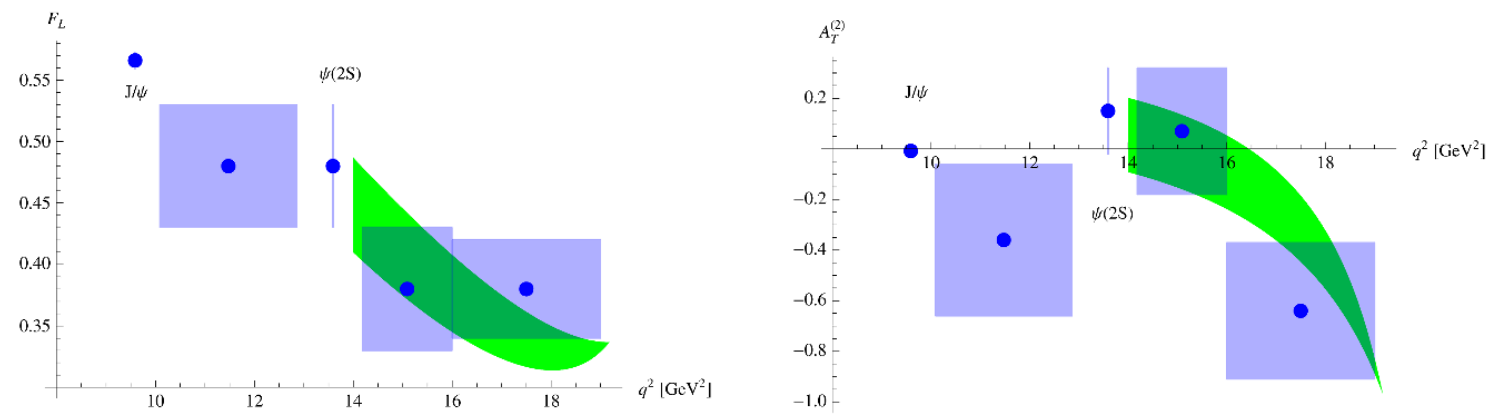

Figure 2. $F_{L}$ (left-hand side) and $A_{T}^{(2)}$ (right-hand side) in the intermediate and low recoil region in $B \rightarrow K^{*} \ell^{+} \ell^{-}$decays on and off $c \bar{c}$-resonance, see table 3 . The (green) band corresponds to a model-independent prediction based on the leading OPE within the SM basis from ref. [9], see text.

we use the OPE at leading order in $1 / m_{b}$ for which $F_{L}$ and $A_{T}^{(2)}$ are form factor based [7]. Under these assumptions ratios of form factors can be obtained from the data and used to predict $F_{L}$ and $A_{T}^{(2)}$. The (green) band corresponds to such a recent determination [9]. The plots are made with the 'SE2 LEL' fit at $1 \sigma$; other parameterisations give comparable results at low recoil, see [9] for details.

For $F_{L}$ and $A_{T}^{(2)}$ the OPE predictions agree well at current precision. This supports that non-factorizable, non-universal contributions are subdominant at low recoil. (For estimations of OPE uncertainties, see [4,39].) As argued in the previous section the increase of $F_{L}$ away from the endpoint region is in the ballpark of what can be expected by the $\kappa$-expansion. Moreover the same is true for extrapolated large recoil factorization results [7], suggesting that non-factorizable (non-universal) contributions are subdominant in ratios at larger recoil as well.

Two further remarks on resonances are in order. First the $\chi_{c 1}$ as well as $\chi_{c 0}$ and $\chi_{c 2}$ are known to receive large non-factorizable corrections and one therefore cannot expect extrapolations from the endpoint to be trustworthy. This is indeed reflected in a rather high value of $F_{L}\left(\chi_{c 1}\right)$, see table 3 . Second, in general observables for resonances cannot be expected to be close to their neighbouring off-resonance counterparts since they couple to a different set of operators. The fact that the resonant $c \bar{c}$-contributions for $F_{L}$ are almost at par with the non-resonant findings, see figure 2 , is therefore non-trivial from the viewpoint of dynamics and supports the kinematic interpretation in terms of the equivalence theorem. The exception being the endpoint region where the predictions become increasingly exact. For $F_{L}$ and $A_{T}^{(2)}$ this also persists to some degree for lower $q^{2}$, as can be inferred from figure 2 , since by the equivalence theorem and the $V$-A interactions both observables are expected to follow a certain pattern in that region. Further compilation and discussion of $F_{L}$ in the context of the non-leptonic decays to two vector mesons is discussed in section 5.1.

We end with a discussion on $P_{4}^{\prime}$ which figures as well as $F_{L}$ and $A_{T}^{(2)}$ amongst the observ-

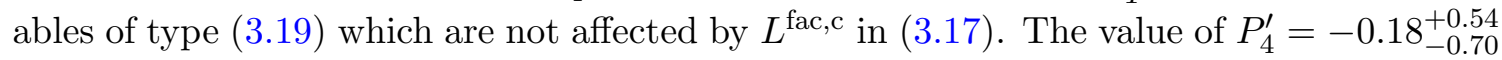
measured in the next-to endpoint bin $[14.18,16] \mathrm{GeV}^{2}[23]$ deviates by $3 \sigma$ and is larger than the expected $\mathcal{O}\left(\kappa^{2} / q^{2}\right)$ away from its endpoint value $\sqrt{2}(3.7)$. This appears to be 
conflicting the conclusions drawn from $F_{L}$ and $A_{T}^{(2)}$ regarding the $\kappa$-expansion and/or the size of the non-factorizable charm contributions although the uncertainties in $P_{4}^{\prime}$ data are currently sizeable. Comparing eqs. (3.20), (3.21) to (3.22), it is tempting to introduce order one phases between $H_{0}$ and $H_{\|}$while keeping ratios of moduli of the HAs approximately unchanged. This choice, however, would affect the ratio $P_{5}^{\prime} / A_{\mathrm{FB}}$, schematically $\propto \operatorname{Re}\left(H_{0} H_{\perp}^{*}\right) / \operatorname{Re}\left(H_{\perp} H_{\|}^{*}\right)$. Its value in the bin $[14.18,16] \mathrm{GeV}^{2}, P_{5}^{\prime} / A_{\mathrm{FB}}=1.66 \pm 0.55[9]$, is in agreement with the endpoint predictions and the value in the endpoint bin, see table 2 . Further measurements of $B \rightarrow K^{*} \ell \ell$ angular observables using different binnings as well as on-resonance measurements of asymmetries can help to clarify these issues and to quantify $L_{\lambda}^{\mathrm{n}-\mathrm{fac}, \mathrm{c}}\left(q^{2}\right)$.

\subsection{Applications to other vector modes}

The endpoint relations obtained for the $B \rightarrow K^{*}(\rightarrow K \pi) \ell^{+} \ell^{-}$-template apply to other modes of the same Lorentz structures and spin configurations. Examples of rare $S \rightarrow V(\rightarrow$ $\left.P_{1} P_{2}\right) \ell^{+} \ell^{-}$decays are

$$
\begin{array}{lll}
B \rightarrow \rho(\rightarrow \pi \pi) \ell^{+} \ell^{-}, & B_{s} \rightarrow \phi(\rightarrow K K) \ell^{+} \ell^{-}, & B_{s} \rightarrow K^{*}(\rightarrow K \pi) \ell^{+} \ell^{-} \\
& B_{c} \rightarrow D_{s}^{*}\left(\rightarrow D_{s} \pi^{0}\right) \ell^{+} \ell^{-}, & B_{c} \rightarrow D^{*}(\rightarrow D \pi) \ell^{+} \ell^{-} \\
& D \rightarrow \rho(\rightarrow \pi \pi) \ell^{+} \ell^{-}, & D_{s} \rightarrow K^{*}(\rightarrow K \pi) \ell^{+} \ell^{-}
\end{array}
$$

as well as lepton flavor violating ones $S \rightarrow V \ell^{+} \ell^{\prime-}$, where $\ell \neq \ell^{\prime}$. The predictions equally apply to the charged current decays $S \rightarrow V\left(\rightarrow P_{1} P_{2}\right) \ell \nu$ :

$$
\begin{array}{rlll}
B \rightarrow D^{*}(\rightarrow D \pi) \ell \nu, & B_{s} \rightarrow D_{s}^{*}\left(\rightarrow D_{s} \pi^{0}\right) \ell \nu, & B_{s} \rightarrow K^{*}(\rightarrow K \pi) \ell \nu, & B \rightarrow \rho(\rightarrow \pi \pi) \ell \nu, \\
& B_{c} \rightarrow \psi(3770)(\rightarrow D D) \ell \nu, & B_{c} \rightarrow D^{*}(\rightarrow D \pi) \ell \nu, \\
& D \rightarrow \rho(\rightarrow \pi \pi) \ell \nu, & D_{(s)} \rightarrow K^{*}(\rightarrow K \pi) \ell \nu, & D_{s} \rightarrow \phi(\rightarrow K K) \ell \nu .
\end{array}
$$

The distributions for the $\ell \nu$ final states differ from the $\ell \ell$ final state by terms of order $\mathcal{O}\left(m_{\ell} / m_{c, b}\right)$. The predictions equally apply to higher states of the above $V$ 's with the same flavor content if kinematically allowed.

\section{$4 \quad B \rightarrow X_{j} \ell \ell$-type decays}

In section 4.1 and section 4.2 we discuss meson decays to spin zero and $\geq 2$, respectively.

\subsection{Spin 0}

The decay $B \rightarrow K \ell \ell$ is of the simplest kind since there is no polarisation tensor for the spin zero $K$. The scalar $\left(O_{S(P)}\right.$ and primed) amplitude $H$ can be non-vanishing as there are simply no Lorentz indices that need to be saturated. The vector $\left(O_{V(A)}\right.$ and primed) amplitudes allow for $\omega^{*}\left(\lambda_{B}\right) \cdot q_{r}$, and hence $H_{\lambda} \propto \mathcal{O}(\kappa)$ for $\lambda=0, \pm 1$ and $H_{t} \propto \mathcal{O}\left(\kappa^{0}\right)$. The latter is accompanied by a factor of $m_{\ell}$ when acting on the lepton-bilinear and one might therefore write $H_{t}^{\text {eff }} \propto \mathcal{O}\left(m_{\ell} \kappa^{0}\right)$. The tensor amplitudes $\left(O_{\mathcal{T}}\right.$ and primed) allow for 
the invariant $\omega_{\mu \nu}^{*}\left(\lambda_{B_{1}}, \lambda_{B_{2}}\right) q_{r}^{\mu} q_{r^{\prime}}^{\nu}$, where $\lambda_{B_{1}}=\bar{\lambda}_{B_{2}}$, by virtue of (2.5), which results in $H_{\lambda \lambda^{\prime}}=0$ and $H_{t \lambda} \propto \mathcal{O}(\kappa)$. In summary we obtain:

$$
H \propto \mathcal{O}\left(\kappa^{0}\right), \quad H_{\lambda} \propto \mathcal{O}(\kappa), \quad H_{t}^{\mathrm{eff}} \propto \mathcal{O}\left(m_{\ell} \kappa^{0}\right), \quad H_{\lambda \lambda^{\prime}}=0, \quad H_{t \lambda} \propto \mathcal{O}(\kappa),
$$

where here $\lambda, \lambda^{\prime}=0, \pm 1$. The power-counting is in agreement with explicit calculations at large and low recoil, $[8,40]$, respectively.

The decay rate $\bar{B} \rightarrow \bar{K} \ell^{+} \ell^{-}$, see eq. (C.1) and footnote 4 for normalization, for a general dimension six operator basis can be written as [40]

$$
\frac{1}{\kappa \beta_{\ell}} \frac{d^{2} \Gamma}{d \cos \theta_{\ell} d q^{2}}=a_{\ell}+b_{\ell} \cos \theta_{\ell}+c_{\ell} \cos \theta_{\ell}^{2}
$$

where the lepton angle $\theta_{\ell}$ is defined as in $\bar{B} \rightarrow \bar{K}^{*} \ell^{+} \ell^{-}$decays. The relations (4.1) imply $b_{\ell}, c_{\ell}\left(q_{\max }^{2}\right)=0$ at the endpoint (here $\left.q_{\max }^{2}=\left(m_{B}-m_{K}\right)^{2}\right)$ and imply isotropicity:

$$
\frac{d^{2} \Gamma}{d \cos \theta_{\ell} d q^{2}} /\left(\frac{d \Gamma}{d q^{2}}\right)=\kappa \beta_{\ell}\left(a_{\ell}+b_{\ell} \cos \theta_{\ell}+c_{\ell} \cos \theta_{\ell}^{2}\right) /\left(\frac{d \Gamma}{d q^{2}}\right) \rightarrow \frac{1}{2},
$$

where $d \Gamma / d q^{2}=\kappa \beta_{\ell} 2\left(a_{\ell}+c_{\ell} / 3\right)$. Eq.(4.3) is also consistent with:

$$
\begin{aligned}
& F_{H}\left(q_{\text {max }}^{2}\right)=\kappa \beta_{\ell} 2\left(a_{\ell}+c_{\ell}\right) /\left(\frac{d \Gamma}{d q^{2}}\right)=1, \\
& A_{\mathrm{FB}}\left(q_{\max }^{2}\right)=\kappa \beta_{\ell} b_{\ell} /\left(\frac{d \Gamma}{d q^{2}}\right) \quad=0 .
\end{aligned}
$$

$F_{H}$ is called flat term in the distribution. We note that (4.1) implies $\left.a_{\ell}\left(q_{\max }^{2}\right)\right|_{\mathrm{SM}}=0$. Non-vanishing endpoint contributions arise from (pseudo-) scalar operators only.

The endpoint relations for HAs and angular distribution for $B \rightarrow K_{0}^{*}(1430) \ell \ell$ decays hold analogously with $C_{+} \rightarrow C_{-}$since $J^{P}\left(K_{0}^{*}\right)=0^{+}$. The decay with $K_{0}^{*}(1430) \rightarrow K \pi$ has been considered as a background process for $B \rightarrow K^{*}(\rightarrow K \pi) \ell \ell$ decays with different angular decomposition [41]. Fortunately, for the low recoil region this effect is small since the kinematic endpoint $q_{\max }^{2}=\left(m_{B}-m_{K_{0}^{*}(1430)}\right)^{2} \simeq(3.85 \mathrm{GeV})^{2}$ barely overlaps with the signal region above $m_{\Psi(2 S)}^{2}$.

Comparing the $\kappa$-counting in eq. (4.1) for $B \rightarrow K \ell \ell$ with the one for $B \rightarrow K^{*} \ell \ell$ in table 1 , one observes that they are different, e.g. $H_{\lambda}^{B \rightarrow K} \propto \mathcal{O}(\kappa)$ whereas $H_{\lambda}^{B \rightarrow K^{*}} \propto \mathcal{O}\left(\kappa^{0}\right)$. On the other hand, there is a relation between $B \rightarrow K \ell \ell$ and decays to a longitudinally $K^{*}$, $B \rightarrow K_{L}^{*} \ell \ell$ at low $q^{2}$ to leading twist and assuming that one of the chiralities dominates, such as in the SM (cf. section 5.1.3. [42]). The absence of a relation at high $q^{2}$, where the twist expansion does not make sense a priori anyway, can also be understood from the fact that at the endpoint there is a democracy amongst the helicity directions and since the $K$-meson has not got any analogue of the \pm 1 helicity direction the relation has got to break down.

\subsection{Higher spin}

We consider decays $S \rightarrow X_{j} \ell^{+} \ell^{-}$(and $S \rightarrow X_{j} \ell \nu$ ), where $X_{j}$ denotes a hadron of spin $J$. For a $S \rightarrow X_{J} X_{J}^{\prime}$-type decay the longitudinal i.e. 0-helicity polarisation fraction assumes 
the value $F_{L}=1 /(2 J+1)[3]$, provided the HAs do not vanish by a selection rule. The situation for $S \rightarrow X_{j} \ell^{+} \ell^{-}$in the effective Hamiltonian approach (2.14) is entirely different.

These statements are illustrated through the $B \rightarrow K_{2}^{*}(1430) \ell^{+} \ell^{-}$-decay. We follow the same type of analyses as in section 2.2, restricting ourselves to the SM + SM' basis. Through invariants: we construct an invariant out of a spin 2 and 1 polarisation tensor $\omega_{\mu \nu}^{*}\left(\bar{\lambda}_{C}\right)$ and $\omega_{\mu}^{*}\left(\lambda_{B}\right)$ as well as $\left(q_{r}\right)_{\mu} \propto \omega_{\mu}^{*}(t)$. The only possible candidate is $\left(P_{1}\right)_{\lambda_{B} \lambda_{C}}=\omega_{\mu \nu}^{*}\left(\bar{\lambda}_{C}\right) \omega^{* \mu}\left(\lambda_{B}\right) \omega^{* \nu}(t)$, which vanishes at the endpoint because of transversity $q_{r} \cdot \omega\left(\lambda_{R}, q_{r}\right)=0$. More precisely one finds that:

$$
H_{\lambda} \propto\left(P_{1}\right)_{\lambda \lambda} \propto \mathcal{O}(\kappa), \quad H_{t} \propto\left(P_{1}\right)_{t 0} \propto \mathcal{O}\left(\kappa^{2}\right)
$$

where $\lambda=0, \pm$ and we have used that $0=\lambda_{A}=\lambda_{B}+\bar{\lambda}_{C}$. Through CGC: the following product is of relevance: $\left(\mathbf{2}_{B} \times \mathbf{1}_{C}\right) \times \mathbf{1}_{q_{r}}=1 \cdot \mathbf{1}+\ldots$ which implies that $H_{\lambda} \propto C_{0 \lambda \bar{\lambda}}^{121}$. Either method yields the following ratios between HAs:

$$
H_{\overline{2}}: H_{\overline{1}}: H_{0}: H_{1}: H_{2}=0: 1: \frac{-2}{\sqrt{3}}: 1: 0 .
$$

It is clear that the corresponding uniangular angular distribution is not isotropic at the endpoint. From (4.6) one obtains $F_{L}\left(q_{\max }^{2}\right)=2 / 5$ and not $1 / 5$ which would be consistent with isotropicity. The fact that $H_{\lambda} \sim \mathcal{O}(\kappa)$ and $H_{t} \sim \mathcal{O}\left(\kappa^{2}\right)(4.5)$ can be seen from [43] at the level of form factor contributions. Taking the endpoint limit in [43] we find agreement with (4.6) up to sign differences which could be due to different conventions of polarisation vectors.

Generalisations of this result are evident. For example for integer $J \geq 2$ and with vector operators $O_{V(A)}$-transition operators, the $H_{\lambda} \propto \mathcal{O}\left(\kappa^{J-1}\right)$ (with $H_{\lambda}=0$ for $|\lambda|>1$ ) and $H_{t} \propto \mathcal{O}\left(\kappa^{J}\right)$. The former can also be understood in another way. Since the $\left(X_{j} \ell \ell\right)$ state is at least in a $l=J-1$-wave (with $l$ being the total orbital angular momentum) the result $H_{\lambda} \propto \kappa^{J-1}$ is therefore expected. The generalisation to higher dimensional operators with higher derivatives is not straightforward because of the timelike polarisation.

\section{Endpoint relations and non-leptonic decays}

In this section we extend the discussion of the introduction to what extent endpoint relations apply to non-leptonic decays. A few sample decay modes are listed at the end of this section.

A hybrid case between the non-leptonic and the semileptonic case arises when the lepton pair is produced via a hadron, e.g., $B_{s} \rightarrow \phi(1680) \Psi(2 S)(\rightarrow \ell \ell)$ for which we expect endpoint symmetries to work. Note that the masses are tuned $\left[\left(m_{B_{s}}, m_{\phi(1680)}, m_{\Psi(2 S)}\right)=\right.$ $(5.36,1.68,3.68) \mathrm{GeV}]$ such that the leptons are likely to be coming from the hadronic state. The decay $B \rightarrow K^{*} \Psi(2 S)$, which is in the wider vicinity of the endpoint cf. figure 2, gives some support of this statement since $F_{L}$ is reasonably close to its endpoint value of $1 / 3$. The essence of these examples are that the production mechanism of the lepton pair is immaterial and it is the quantum numbers of the actual final states which determine the helicity structure at and near the endpoint. 


\begin{tabular}{|l||lllll|}
\hline$B \rightarrow V_{1} V_{2}$ & $B^{0} \rightarrow D_{s}^{*+} D^{*-}$ & $B^{0} \rightarrow J / \Psi K^{* 0}$ & $B^{0} \rightarrow D^{*+} D^{*-}$ & $B^{0} \rightarrow D_{s}^{*+} \rho^{-}$ & $B^{0} \rightarrow \rho^{+} \rho^{-}$ \\
\hline$u$ & 0.61 & 0.57 & 0.58 & 0.28 & 0.09 \\
$F_{L}[10]$ & $0.52 \pm 0.06$ & $0.570 \pm 0.008$ & $0.624 \pm 0.031$ & $0.84 \pm 0.03$ & $0.977 \pm 0.026$ \\
\hline
\end{tabular}

Table 4. Selected data on $B \rightarrow V_{1} V_{2}$ decays that illustrate $\left.F_{L}\right|_{u=1}=1 / 3$ and $\left.F_{L}\right|_{u \rightarrow 0} \rightarrow 1$. Whereas the former is exact the latter limit is based on qualitative arguments which can be invalidated by the specific dynamics, see text.

What happens in a decay which is purely non-leptonic such as $B \rightarrow K^{*}(\rightarrow K \pi) p \bar{p}$ for example is more complicated as in general one expects virulent final state interactions. Consider that the decay $B \rightarrow K^{*} p \bar{p}$ proceeds as depicted in figure 1 (with $A=B, C=K^{*}$ and $\left.B_{1,2}=p, \bar{p}\right)$, then there is a configuration where $\kappa=\left|\vec{q}_{K^{*}}\right|=0$ which implies $\vec{q}_{p}=-\vec{q}_{\bar{p}}$ for which we can expect isotropicity (in $\theta_{p}$ ). The crux is though that the $K^{*}$ then decays into $K \pi$ and that these particles will interact strongly with the $p \bar{p}$-pair. In the extreme case of inelasticity this leads to a different final state (say $p K \rightarrow \Sigma \pi$ ) or the $K$ and the $p$ might simply exchange momentum, both of which changes the kinematics in general. Thus we are led to conclude that endpoint symmetry holds, if one restricts oneself to the subset of decay configurations from $B \rightarrow K^{*}(\rightarrow K \pi) p \bar{p}$ decays for which $\vec{q}_{p}=-\vec{q}_{\bar{p}}$. The latter could be realized if the proton pair does not interact significantly with the $K^{*}$-meson. In essence we expect endpoint symmetries to play a rôle on a subset of configurations which is defined by $\vec{q}_{C}=0$ and $q_{C}^{2}=m_{C}^{2}$.

Decays that could be studied experimentally consist of $\left(B_{1} B_{2}\right)$ pairs which either scalars such as $(\pi \pi),(\pi K)$ or long-lived spin-1/2 baryons such as $(p \bar{p})$ or $(\Lambda \bar{\Lambda})$. For the baryons several modes have been observed to date, $B \rightarrow D^{0 *} p \bar{p}[44], B \rightarrow K^{*} \Lambda \bar{\Lambda}$ [45] and $B \rightarrow K^{*} p \bar{p}$. For the latter there are already $K^{*}$-polarisation measurements, which however do not cover the endpoint region [46]. The decay $B_{(s)} \rightarrow J / \Psi p \bar{p}$ has been searched for at LHCb [47]. Corresponding branching ratios are $\mathcal{B}\left(B \rightarrow K^{*} \Lambda \bar{\Lambda}, K^{*} p \bar{p}\right)=$ few $\times \mathcal{O}\left(10^{-6}\right)$ and $\mathcal{B}\left(B \rightarrow D^{0 *} p \bar{p}\right)=\mathcal{O}\left(10^{-4}\right)[10]$.

\subsection{Hadronic $S \rightarrow V_{1} V_{1}$ and qualitative remarks on $F_{L}$}

Decays of the type $S \rightarrow V_{1} V_{2}$ are generically not in an endpoint configuration. Here we take the quantity $u \equiv\left(\left(m_{V_{1}}+m_{V_{2}}\right) / m_{S}\right)^{2}$ as a measure of the distance from the endpoint. Towards the endpoint $\left.F_{L}\right|_{u \rightarrow 1} \rightarrow 1 / 3$ (3.4) (with available phase space vanishing), and naively we expect $\left.F_{L}\right|_{u \rightarrow 0} \rightarrow 1$ by virtue of the equivalence theorem as explained in appendix D. The difference to the discussion in the appendix is though that neither of the vector mesons is observed through $V \rightarrow \gamma^{*} \rightarrow \ell \ell$ and the cancellations leading to the zero of the $A_{\mathrm{FB}}$ are therefore absent. In table 4 we collect data on selected $B \rightarrow V_{1} V_{2}$ decays which support the anticipated pattern in $F_{L}(u)$.

Specific dynamics including $V+A$ admixture can, however, invalidate the kinematic picture. For example $F_{L}\left(B^{0} \rightarrow \phi K^{* 0}\right)_{u=0.13}=0.480 \pm 0.030$ [10] differs considerably from $F_{L}\left(B^{0} \rightarrow \rho^{+} \rho^{-}\right)_{u=0.09}=0.977 \pm 0.026$ despite their close $u$-values. An explanation was pointed out in $[48]^{6}$ where it was observed that in penguin dominated decays formally

\footnotetext{
${ }^{6}$ For a more complete classification we refer the reader to reference [49].
} 


\begin{tabular}{|l||ccccc|}
\hline$D \rightarrow V_{1} V_{2}$ & $\rho \rho$ & $K^{*} K^{*}$ & $K^{*} \rho$ & $\phi \rho$ & $\phi K^{*}$ \\
\hline$u\left(D_{0}, D_{ \pm}\right)$ & 0.68 & 0.92 & 0.80 & 0.92 & - \\
$u\left(D_{s}\right)$ & 0.61 & 0.83 & 0.72 & 0.83 & 0.95 \\
\hline
\end{tabular}

Table 5. $u$-values for $D \rightarrow V_{1} V_{2}$ decays. The columns (rows) correspond to final (initial) states.

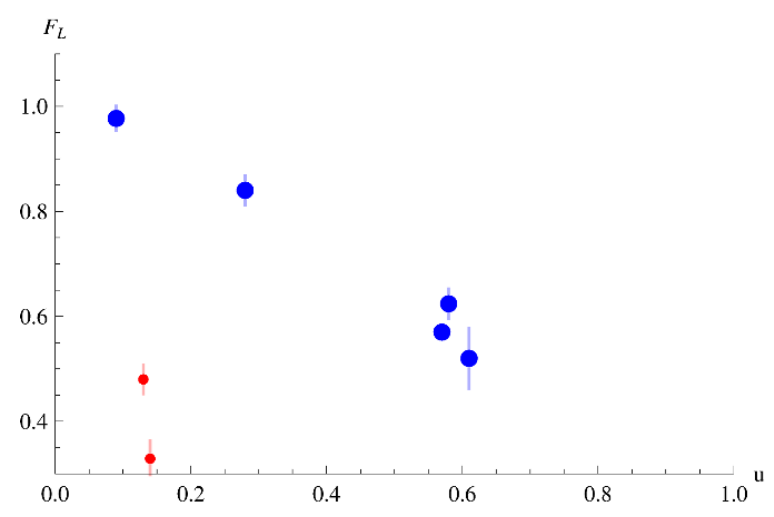

Figure 3. $F_{L}$ for $B \rightarrow V_{1} V_{2}$ examples (blue points) for $\left.F_{L}\right|_{u=1}=1 / 3$ and $\left.F_{L}\right|_{u \rightarrow 0} \rightarrow 1$, as in table 4 . The smaller (red) points correspond to decays with different weak dynamics, see text.

subleading contributions in $1 / m_{b}$, known as weak annihilation, are numerically large and can in principle accommodate the experimental results. Other examples of the same kind, recently measured by the LHCb collaboration, are $F_{L}\left(B_{s} \rightarrow \phi \phi\right)_{u=0.14}=0.329 \pm 0.033 \pm$ 0.017 [50] and $F_{L}\left(B_{s} \rightarrow \phi K^{*}\right)_{u=0.13}=0.51 \pm 0.15 \pm 0.07$ [51]. In figure 3 we show the decays from table 4 supporting the kinematic $F_{L}$-pattern (blue points) as well as the rather precisely measured penguin modes where it does not work (smaller red points).

The uncertainties of the weak annihilation theory predictions are rather large in QCD factorization because of endpoint (infrared) divergences for which a cut-off has to be introduced. This precludes conclusions on possible new physics signals in those observables. Further analysis is beyond the scope of this paper.

Predictions for $D \rightarrow V_{1} V_{2}$ decays are more stable as the corresponding $u$-values are larger and in some cases very close to the endpoint kinematics, see table 5 . While current data on $F_{L}$ in these modes except for $F_{L}\left(D^{0} \rightarrow \rho^{0} \rho^{0}\right)=0.690 \pm 0.074$ [10] are not precise enough for a comparison with the endpoint prediction and asymptotics, we encourage further study.

\section{Summary}

The key results of this work are the endpoint relations for the helicity amplitudes (2.15) and angular observables of a decaying spinless particle into a vector meson and two leptons, eq. (3.2). Constraints on observables are summarised in eqs. (3.3)-(3.9). The physics origin of the relations is the absence of direction at the point of zero recoil. The helicity relations apply analogously to charged-current decays (3.25), including for example $B \rightarrow D^{*} \ell \nu$. In the case of di-neutrino modes $B \rightarrow K^{*} \nu \bar{\nu}$ [26] and all modes with $\ell=\nu$ from eq. (3.24) 
$F_{L}=1 / 3$ constrains the shape of the decay distribution. As the symmetry is independent of the dynamics of the decay but rather depends on the spin of the external states, it applies as well to non-leptonic decays of the type $B \rightarrow K^{*} p \bar{p}$ provided that the $K^{*}$ does not interact significantly with the proton pair. We investigated the vicinity of the endpoint through an expansion in the three momentum of the $V$-meson in the $B$-cms in section 3.2.

The endpoint relations are beneficial in guiding experimental searches. Existing endpoint data is found to be consistent with the endpoint symmetries, see table 2 . The agreement validates that backgrounds are under control. In addition, eq. (3.4) can be used to remove backgrounds from other resonant (and non-resonant) contributions to $S \rightarrow P_{1} P_{2} \ell^{+} \ell^{-}$ from intermediate particles other than spin 1 . An important example is the $\mathrm{S}$-wave contributions to $B \rightarrow K \pi \ell^{+} \ell^{-}$, recently addressed in refs. $[41,52,53]$ for the low $q^{2}$ region.

New physics opportunities arise from in $d \Gamma / d \phi$, eq. (3.5), the distribution between the two final decay planes, which is sensitive to tensor operator contributions, as well as the slopes in the vicinity of the endpoint. The latter, denoted by $\hat{R}, \hat{I}$ in (3.11), (3.12), are universal and appear in several observables. The overdetermination of $\hat{R}$ and $\hat{I}$ provides a model-independent check. Current extractions from e.g., $A_{\mathrm{FB}}$ and $P_{5}^{\prime}$ are consistent with each other and with the SM. Second order corrections in the momentum expansion are not universal but for observables like $F_{L}, A_{T}^{(2)}$ and $P_{4}^{\prime}$, in which resonance effects mostly drop out (cf. section 3.3.1), the relative change with respect to the endpoint prediction serves as an additional test. While data on $F_{L}$ and $A_{T}^{(2)}$ change only moderately and accordingly in the next to endpoint bin, $P_{4}^{\prime}[23]$ deviates substantially and escapes interpretations within factorization $[9,54-56]$. It will be interesting to see how this develops with the forthcoming analysis of LHCb's $3 \mathrm{fb}^{-1}$ data set, and with the future data taking of the Belle II experiment.

Additionally our findings explain the observed universal helicity structure of $B \rightarrow$ $K^{*} \ell^{+} \ell^{-}$, and the ones in (3.24), in the SM basis [7] (cf. appendix B) manifest in the low recoil OPE [4]. Furthermore we found that $F_{L}$ follows a kinematic pattern imposed by the endpoint symmetry and the equivalence theorem (cf. appendix D), at the exception of channels with numerically enhanced weak annihilation contributions, see figure 3 . The corresponding data on non-leptonic decays is compiled and commented on in section 5.1. We investigated several generalisations, including decays into a spin-zero and spin-2 meson plus two fermions. Further decays maybe studied at and in the vicinity of their endpoint, towards a more precise interpretation of future weak decay data.

\section{Acknowledgments}

We are grateful to Greig Cowan, Markus Hopfer, Sebastian Jäger, Jürgen Körner, James Lyon, Franz Muheim, Matt Needham, Stefan Schacht and Wei Wang for useful discussions. RZ acknowledges the support of an advanced STFC fellowship. This work is supported by the Deutsche Forschungsgemeinschaft (DFG) within research unit FOR 1873. 


\section{A Conventions for polarisation vectors}

Our conventions of the physical polarisation tensors are those of Jacob and Wick [1] and are the same as in [3]. For a polarisation vector for a particle at rest we have:

$$
\omega( \pm)=(0, \pm 1, i, 0) / \sqrt{2}, \quad \omega(0)=(0,0,0,1) \quad \text { and } \quad \omega(t)=(1,0,0,0) .
$$

Throughout this work we use $(+,-) \leftrightarrow(1, \overline{1}) \leftrightarrow(1,-1)$ interchangeably for a spin 1 polarisation index. The identification with the polarisation tensors $\alpha, \beta, \gamma$ of the particles $A, B$ and $C$, respectively, taken to be $J=1$, is as follows:

$$
\begin{gathered}
\omega( \pm)=\alpha( \pm)=\beta( \pm)=\gamma(\mp), \\
\omega(0)=\alpha(0)=\beta(0)=\gamma(0),
\end{gathered}
$$

where the last two equalities are valid at the endpoint as can be seen from the explicit parameterisation to given below. The scalar products are

$$
\omega\left(\lambda_{1}\right) \cdot \omega^{*}\left(\lambda_{2}\right)=-\delta_{\lambda_{1} \lambda_{2}}, \quad \omega\left(\lambda_{1}\right) \cdot \omega\left(\lambda_{2}\right)=-(-1)^{\lambda_{1}} \delta_{\lambda_{1} \bar{\lambda}_{2}} .
$$

The only complication is that the quantization axis of the $C$-particle is opposite to the ones of the $A$ - and $B$-particles. This implies a helicity flip as indicated in the equation above. Furthermore in the Jacob-Wick phase convention the helicity dependent phase is chosen to be unity $[1,2]$. We emphasise that the conventions (A.1) are consistent with the Condon-Shortly phase convention which are standard for the CGC [10].

The power counting of the $\kappa$-corrections in the vicinity of the endpoint in the decay $A \rightarrow\left(B_{1} B_{2}\right) C$ can be verified by using the explicit parameterisation of the vectors in the $A$-cms:

$$
\begin{array}{lll}
q_{B}=q=\left(\left(q_{B}\right)_{0}, 0,0, \kappa\right), & \beta(0)=\left(\kappa, 0,0,\left(q_{B}\right)_{0}\right) / \sqrt{q_{B}^{2}}, & \beta(t)=q_{B} / \sqrt{q_{B}^{2}}, \\
q_{C}=p=\left(\left(q_{C}\right)_{0}, 0,0,-\kappa\right), & \gamma(0)=\left(-\kappa, 0,0,\left(q_{C}\right)_{0}\right) / \sqrt{q_{C}^{2}}, & \gamma( \pm)=\beta(\mp)=\omega(\mp) .
\end{array}
$$

Above $\left(q_{B}\right)_{0} \equiv \sqrt{q_{B}^{2}+\kappa^{2}},\left(q_{C}\right)_{0} \equiv \sqrt{q_{C}^{2}+\kappa^{2}}=m_{A}-\left(q_{B}\right)_{0}$ and the physical polarisation vectors are transverse $q_{B} \cdot \beta(\lambda)=0, q_{C} \cdot \gamma(\lambda)=0$ for $\lambda=0$, \pm . For assessing the $\kappa$ expansion we choose the $A$ - and $C$-particle to be on-shell $q_{A}^{2}=m_{A}^{2}$ and $q_{C}^{2}=m_{C}^{2}$. The variable $q_{B}^{2}=q_{\max }^{2}+\mathcal{O}\left(\kappa^{2}\right)$ is $\kappa$-dependent through eq. (2.17). The distance to the kinematic endpoint is $\delta q^{2} \equiv\left(m_{A}-m_{C}\right)^{2}-q_{B}^{2}=\mathcal{O}\left(\kappa^{2}\right)$. With these conventions the relevant scalar products read:

$$
\begin{aligned}
& \beta(0) \cdot q_{C}=\kappa m_{A} / \sqrt{q_{B}^{2}}=\mathcal{O}(\kappa) \\
& \beta( \pm) \cdot q_{C}=0 \\
& \beta(t) \cdot q_{C}=\left(m_{A}^{2}-q_{B}^{2}-m_{C}^{2}\right) /\left(2 \sqrt{q_{B}^{2}}\right)=\mathcal{O}\left(\kappa^{0}\right)
\end{aligned}
$$


and

$$
\beta\left(\lambda_{B}\right) \cdot \gamma\left(\lambda_{C}\right)= \begin{cases}1 & \lambda_{B}=\bar{\lambda}_{C}= \pm \\ \left(-\kappa^{2}-\sqrt{\kappa^{2}+q_{B}^{2}} \sqrt{\kappa^{2}+m_{C}^{2}}\right) /\left(\sqrt{q_{B}^{2}} m_{C}\right) & \lambda_{B}=\lambda_{C}=0 \\ 0 & \text { otherwise }\end{cases}
$$

\section{B The low recoil OPE and non-factorizable corrections in the light of endpoint relations}

It was observed that in the SM+SM' basis the effective Wilson coefficients (EWC) $C_{ \pm}^{\mathrm{eff}}\left(q^{2}\right) \equiv C^{\mathrm{eff}}\left(q^{2}\right) \pm C^{\mathrm{eff}^{\prime}}\left(q^{2}\right)$ are independent of the $V$ mesons' polarisation [7, 8, 57]. In the transversity basis this amounts to

$$
\begin{array}{ll}
B \rightarrow V \ell \ell: & H_{0, \|}=C_{-}^{\mathrm{eff}}\left(q^{2}\right) f_{0, \|}\left(q^{2}\right), \quad H_{\perp}=C_{+}^{\mathrm{eff}}\left(q^{2}\right) f_{\perp}\left(q^{2}\right), \\
B \rightarrow \text { P } \ell: & H=C_{+}^{\mathrm{eff}}\left(q^{2}\right) f\left(q^{2}\right),
\end{array}
$$

where $f_{i}(i=0, \perp, \|)$ are the usual polarisation-dependent $B \rightarrow V$ form factors and $f$ denotes the corresponding $B \rightarrow P$ form factor. Generally the expression (B.1) is subject to non- factorizable corrections

$$
f_{\lambda}\left(q^{2}\right) \rightarrow f_{\lambda}\left(q^{2}\right)\left(1+\epsilon_{\lambda}\left(q^{2}\right)\right), \quad \epsilon_{\lambda}\left(q^{2}\right)=\mathcal{O}\left(\alpha_{s} / m_{b},\left[\mathcal{C}_{7} / \mathcal{C}_{9}\right] / m_{b}\right), \quad \lambda=0, \pm 1 .
$$

We would like to point out that the endpoint relation imply degeneracy of the corrections at the endpoint

$$
\epsilon_{\lambda}\left(q_{\max }^{2}\right) \equiv \epsilon, \quad \lambda=0, \pm 1, \|, \perp,
$$

with (2.15) already enforced by the form factors $f_{\lambda}, f_{\perp}\left(q_{\max }^{2}\right)=0$ and $f_{\|}\left(q_{\max }^{2}\right)=$ $\sqrt{2} f_{0}\left(q_{\max }^{2}\right)$ as used e.g., in ref. [9]. Below we give two alternative viewpoints on why the EWC are polarisation independent in the low recoil region.

- For the true Wilson coefficients, which in particular are $q^{2}$ - independent universality follows from factorization, a property of the OPE. The points to be discussed are contributions from off-shell photons and quark loops e.g., $\bar{b} s \bar{c} c$-operators which lead to so-called charm loops. For the former the improved Isgur-Wise relations [5, 6] are instrumental, which render the relative size of the electromagnetic dipole to (axial)vector operator contributions universal. The origin of this feature are the QCD equations of motions, further discussed in [9]. The reason why (B.1) holds for the charm loops as well is that those loops factorize and do therefore not introduce a new dependence on the polarisation per se. More precisely the charm loop corresponds to the vacuum polarisation which is proportional to $g_{\alpha \beta}$ and $q_{\alpha} q_{\beta}$ structures where $q$ is the external momentum going through the loop. The latter structure vanishes when it is propagated by the photon to a vector current by the conservation of the latter. The $g_{\alpha \beta}$-structure does not change the polarisation. Thus the factoriztion into a form factor and direct evaluation of a $\bar{\ell} \gamma_{\mu} \ell$ matrix element implies (B.1). 
- Another viewpoint is that eq. (2.15) enforces within (B.1) relations between the form factors. To make this explicit consider a generic ansatz as

$$
H_{\lambda}=C_{\lambda}\left(q^{2}\right) f_{\lambda}\left(q^{2}\right)+C_{\lambda}^{\prime}\left(q^{2}\right) f_{\bar{\lambda}}\left(q^{2}\right), \quad \lambda=0, \pm 1 .
$$

By virtue of parity covariance (cf. table 1 ) the form factors obey $f_{\lambda}^{\prime}=-f_{\bar{\lambda}}$. Since the form factors obey the same endpoint relations as the amplitudes $(2.15) f_{0}\left(q_{\max }^{2}\right)=$ $-f_{1}\left(q_{\max }^{2}\right)=-f_{\overline{1}}\left(q_{\max }^{2}\right)$ it follows that the combination $C_{\lambda}\left(q_{\max }^{2}\right)-C_{\lambda}^{\prime}\left(q_{\max }^{2}\right)=C$ is polarisation independent. Moreover, since the relation must hold irrespective of the ratio of $V-A$ and $V+A$ operators this implies complete degeneracy at the endpoint. One might wonder whether the equality of the EWC holds at low recoil as well as at the endpoint. The answer to that is affirmative as the EWC does not know about the location of the endpoint. We could for instance apply the formalism to $B \rightarrow K^{*}(1410) \ell \ell$ in which case the endpoint would lower from $\left(m_{B}-m_{K^{*}}\right)$ to $\left(m_{B}-m_{K^{*}(1410)}\right)$ with the same EWC. Thus one gets

$$
H_{\lambda}=C\left(q^{2}\right) f_{\lambda}\left(q^{2}\right)-C^{\prime}\left(q^{2}\right) f_{\bar{\lambda}}\left(q^{2}\right),
$$

which is equivalent to the expression in (B.1) after identifying $C^{\left({ }^{\prime}\right)}\left(q^{2}\right)$ with $C^{\text {eff(') }}\left(q^{2}\right)$.

At last we would like to point out that the results (B.1) are valid independent of the chirality of the lepton interaction vertex. One could restore $H_{i} \rightarrow H_{i}^{L, R}$ with $L, R$ referring to $\ell \rightarrow \ell_{L, R}$. The fact that in general the Wilson coefficient of the transverse HA is different from the other can also be inferred from the fact that the corresponding form factor vanishes at the endpoint $f_{\perp}\left(q_{\max }^{2}\right)=0$ and evades a constraint. For models with $|C| \gg\left|C^{\prime}\right|$, such as the SM, degeneracy in all transversity directions $0, \|, \perp$ is effectively attained.

\section{Phenomenological parameterisations for helicity amplitudes}

Within the SM + SM' basis (neglecting $H_{t}$ which is suppressed by $m_{\ell}$ ) one may parameterise $H_{\lambda}, \lambda=0, \|, \perp$ to be used in fits to experimental data. We suggest here a phenomenological, local parameterisation adapted to low and high $q^{2}$ without working out all details. ${ }^{7}$

The $B \rightarrow V \ell \ell$ decay rate scales like $d \Gamma \propto \kappa \kappa_{\ell} d q^{2} d$ (angles) where $\kappa$ and $\kappa_{\ell}$ denotes the absolute value of the three momentum of the $K^{*}$ and $\ell$-particles, respectively. The former is given in eq. (2.17) and the latter is given by

$$
\kappa_{\ell} \equiv \sqrt{\frac{\lambda\left(q^{2}, m_{\ell}^{2}, m_{\ell}^{2}\right)}{4 q^{2}}}=\frac{1}{2} \sqrt{q^{2}} \beta_{\ell}, \quad \beta_{\ell} \equiv \sqrt{1-4 \frac{m_{\ell}^{2}}{q^{2}}} .
$$

In counting and parameterising higher orders in $\kappa^{2}$ or $q^{2}$ it is therefore important to keep track of both $\kappa$ and $\kappa_{\ell}$. In the conventions of reference [8] this is taken care of by a global factor $\sqrt{\kappa \kappa_{\ell}}$ in the HAs. Below we do not write the $\sqrt{\kappa \kappa_{\ell}}$-prefactor explicitly.

\footnotetext{
${ }^{7}$ One needs to take into account that the angular coefficients $J_{i}$ are bilinears of the HAs. This leads to a U(2)-symmetry and reduces the number of free parameters by four [58].
} 


\section{C.1 Low recoil - high $q^{2}$}

We extend the $\kappa$-expansion (3.10) to:

$$
\begin{aligned}
-\sqrt{2} H_{0}^{x} & =\sqrt{q_{\max }^{2} / q^{2}}\left(a_{0}^{x}+b_{0}^{x} \kappa^{2}+\frac{c_{0}}{q^{2}}+\ldots\right)\left(1+\sum_{r} \Delta_{0}^{(r)}\left(q^{2}\right)\right), \quad x=L, R, \\
H_{\perp}^{x} & =\kappa\left(a_{\perp}^{x}+b_{\perp}^{x} \kappa^{2}+\frac{c_{\perp}}{q^{2}}+\ldots\right)\left(1+\sum_{r} \Delta_{\perp}^{(r)}\left(q^{2}\right)\right), \\
H_{\|}^{x} & =\left(a_{\|}^{x}+b_{\|}^{x} \kappa^{2}+\frac{c_{\|}}{q^{2}}+\ldots\right)\left(1+\sum_{r} \Delta_{\|}^{(r)}\left(q^{2}\right)\right),
\end{aligned}
$$

where the coefficients $a, b$ and $c$ are complex (see, however, footnote 7 ), the function $\Delta^{(r)}\left(q^{2}\right)$ take into account effects of resonances and the ellipses stand for higher orders in $\kappa$. Locally the $\Delta$-function can be approximated by a Breit-Wigner ansatz $\Delta_{i}^{(r)}\left(q^{2}\right)=\delta_{i}\left(q^{2}\right) /\left(q^{2}-m_{r}^{2}+\right.$ $\left.i \Gamma_{r} m_{r}\right)$ where $m_{r}, \Gamma_{r}$ are the corresponding masses and decay widths. In the factorization approximation $\delta_{i}\left(q^{2}\right)=\delta_{i}$ is a constant and deviations thereof are a measure of nonfactorizable contributions.

The endpoint constraint (2.15) implies:

$$
\left(a_{0}^{x}+c_{0} / q_{\max }^{2}\right)\left(1+\sum_{r} \Delta_{0}^{(r)}\left(q_{\max }^{2}\right)\right)=\left(a_{\|}^{x}+c_{\|} / q_{\max }^{2}\right)\left(1+\sum_{r} \Delta_{\|}^{(r)}\left(q_{\max }^{2}\right)\right), \quad x=L, R .
$$

Since the $a, c, \Delta$-terms stem in general from different operators in the effective Hamiltonian, barring fine-tuning, the endpoint relation must be satisfied for each term separately, that is

$$
a_{0}^{x}=a_{\|}^{x}, \quad c_{0}=c_{\|}, \quad \Delta_{0}^{(r)}\left(q_{\max }^{2}\right)=\Delta_{\|}^{(r)}\left(q_{\max }^{2}\right),
$$

consistent with eq. (B.3).

A few remarks are in order. First of all, the parameterisation (C.2) aims at an efficient phenomenological description and is supposed to hold locally, unlike the OPE. The factor $1 / \sqrt{q^{2}}$ in front of $H_{0}$ originates from the normalisation of the polarisation vector (A.4). The resonance terms $c$ as well as $\Delta^{(r)}$ originate from photons and thus couple vector-like, $c^{R}=c^{L}, \Delta^{R}=\Delta^{L}$ and we have therefore suppressed in (C.2) their chirality labels. Instead of $\kappa^{2}$ one could also expand in $\delta q^{2} \equiv\left(q_{\max }^{2}-q^{2}\right)$ cf. (C.7). The deviation of ratios from one, $\left[\Delta_{\lambda}^{(r)}\left(q^{2}\right)\right] /\left[\Delta_{\lambda^{\prime}}^{(r)}\left(q^{2}\right)\right] \neq 1$ is a $q^{2}$-dependent measure of non-factorizable contributions to observables which depend on ratios of HAs.

The remaining HAs that arise in a general dimension six effective Hamiltonian can be parameterised in an analogous but simpler manner as $c \bar{c}$-resonances are absent:

$$
\begin{array}{lll}
H_{X}=\kappa / \sqrt{q^{2}}\left(a_{X}+b_{X} \kappa^{2}+\ldots\right), & \text { for } H_{X}=H_{\perp}^{\mathcal{T}}, H_{\perp}^{\mathcal{T}_{t}}, H_{t}, \\
H_{X}=\sqrt{q_{\max }^{2} / q^{2}}\left(a_{X}+b_{X} \kappa^{2}+\ldots\right), & \text { for } H_{X}=H_{\|}^{\mathcal{T}}, H_{\|}^{\mathcal{T}_{t}}, \\
H_{X}=a_{X}+b_{X} \kappa^{2}+\ldots, & \text { for } H_{X}=H_{0}^{\mathcal{T}}, H_{0}^{\mathcal{T}_{t}}, \\
H=\kappa / \sqrt{q_{\max }^{2}}\left(a+b \kappa^{2}+\ldots\right) . &
\end{array}
$$

Note that the pseudo-scalar contribution is absorbed as usual in the vector one by the equations of motion. 


\section{C.2 Large recoil - low $q^{2}$}

At large recoil a similar parameterisation to the one at low recoil given in the previous subsection can be employed: ${ }^{8}$

$$
\begin{aligned}
-\sqrt{2} H_{0}^{x} & =\sqrt{q_{\max }^{2} / q^{2}}\left(\alpha_{0}^{x}+\beta_{0}^{x} q^{2}+\ldots\right)\left(1+\sum_{r} \Omega_{0}^{(r)}\left(q^{2}\right)\right), \quad x=L, R, \\
H_{\perp}^{x} & =\kappa\left(\alpha_{\perp}^{x}+\beta_{\perp}^{x} q^{2}+\frac{\varphi_{\perp}}{q^{2}} \ldots\right)\left(1+\sum_{r} \Omega_{\perp}^{(r)}\left(q^{2}\right)\right), \\
H_{\|}^{x} & =\left(\alpha_{\|}^{x}+\beta_{\|}^{x} q^{2}+\frac{\varphi_{\|}}{q^{2}}+\ldots\right)\left(1+\sum_{r} \Omega_{\|}^{(r)}\left(q^{2}\right)\right),
\end{aligned}
$$

where $\alpha, \beta$ and $\varphi$ are complex numbers (cf. again footnote 7), the dots stand for higher orders in $q^{2}$ and the functions $\Omega$ take into account resonance structures. Note there is no $\varphi_{0}$-term since the photon does not have a zero helicity component. The very same remarks, as for the $\Delta$-functions in the previous subsection, apply for the $\Omega$-terms. Depending on the region it might be sufficient to approximate them with a Breit-Wigner ansatz: $\Omega_{i}^{(r)}\left(q^{2}\right)=$ $\omega_{i}^{(\rho / \omega)}\left(q^{2}\right) /\left(q^{2}-m_{\rho / \omega}^{2}+i m_{\rho / \omega} \Gamma_{\rho / \omega}\right), \omega_{i}^{(J / \Psi)}\left(q^{2}\right) /\left(q^{2}-m_{J / \Psi}^{2}+i m_{J / \Psi} \Gamma_{J / \Psi}\right)$. Dependence on $q^{2}$ of $\omega$ as well as polarisation dependence are a measure of non-factorizable effects. In fact to some extent the residues $\omega^{(\rho / \omega)}$ and $\omega^{(J / \Psi)}$ can be seen as modelling the effect of a sum of resonances at the cost of the $q^{2}$-dependence which is though not of major impact as long as one does not get too close to the resonances. By restricting the interval away from the $\rho$ - and or $J / \Psi$-resonances one could drop the $\Omega$-terms at the expense of less statistics. Note, the radiation of photons from light quarks, described at $q^{2}=0$ by the photon distribution amplitude, are mimicked at $q^{2}>1 \mathrm{GeV}^{2}$ by $\langle\bar{q} q\rangle / q^{2}$-terms. This leads to $1 / q^{4}$-contributions in $H_{\|, \perp}$ [42] in processes such weak annihilation for example. These contributions are though relatively small in the SM other than for the isospin asymmetry (which is small by itself).

\section{C.3 Three momentum $\kappa$ versus $q^{2}$}

In this appendix we give auxiliary formulae relating $\kappa$ and $q^{2}$. From (2.17) one gets:

$$
\begin{aligned}
\kappa & =\frac{1}{2 m_{B}} \sqrt{\left(q_{\max }^{2}-q^{2}\right)\left(\bar{q}_{\max }^{2}-q^{2}\right)}=\frac{1}{2 m_{B}} \sqrt{\delta q^{2}\left(\delta q^{2}+4 m_{B} m_{K^{*}}\right)} \\
& =\sqrt{\delta q^{2}\left(m_{K^{*}} / m_{B}\right)}\left(1+\frac{\delta q^{2}}{8 m_{B} m_{K^{*}}}-\frac{\left(\delta q^{2}\right)^{2}}{16 m_{B}^{2} m_{K^{*}}^{2}}+\ldots\right),
\end{aligned}
$$

where $\delta q^{2}$ is defined as

$$
\delta q^{2} \equiv q_{\max }^{2}-q^{2}, \quad q_{\max }^{2}\left(\bar{q}_{\max }^{2}\right)=\left(m_{B} \mp m_{K^{*}}\right)^{2},
$$

the (positive) distance to the endpoint in the physical region. Thus to leading order $\kappa^{2} \propto \delta q^{2}$ or more precisely:

$$
\delta q^{2}=2 m_{B} m_{K^{*}}\left(\sqrt{1+\kappa^{2} / m_{K^{*}}^{2}}-1\right)=\frac{m_{B}}{m_{K^{*}}} \kappa^{2}+\mathcal{O}\left(\kappa^{4}\right) .
$$

\footnotetext{
${ }^{8}$ It was suggested to apply $q^{2}$-expansion in the large recoil region $[59,60]$.
} 


\section{D $\quad F_{L}$ asymptotics}

For $B \rightarrow V \ell \ell$ the observable $F_{L}$ interpolates between $F_{L}(0)=0$ where the longitudinal mode decouples completely and $F_{L}\left(q_{\max }^{2}\right)=1 / 3(3.4)$ where all polarisations are equally probable. This raises the question of whether anything can be said about $F_{L}$ in between those two kinematic limits. The zero of the $A_{\mathrm{FB}}$ and the equivalence theorem turn out to be of relevance in this context. For the subsequent analysis it is helpful to rewrite $F_{L}$ and $A_{\mathrm{FB}}$ in the $\lambda=0, \pm 1$ helicity and $V, A$ lepton-chirality basis:

$A_{\mathrm{FB}} \propto \operatorname{Re}\left[H_{-}^{V} H_{-}^{A *}-H_{+}^{V} H_{+}^{A *}\right], \quad F_{L}=\frac{\left|H_{0}^{A}\right|^{2}+\left|H_{0}^{V}\right|^{2}}{\left|H_{0}^{A}\right|^{2}+\left|H_{0}^{V}\right|^{2}+\left|H_{+}^{A}\right|^{2}+\left|H_{+}^{V}\right|^{2}+\left|H_{-}^{A}\right|^{2}+\left|H_{-}^{V}\right|^{2}}$.

The $V$-A nature of the weak interaction distinguishes between the two \pm -helicity directions. In particular for low $q^{2}$ this leads to $H_{+}^{V, A} \simeq 0$ which renders $H_{-}^{V, A}$ at the same time larger by roughly a factor of $\sqrt{2}$. In the same kinematic region the zero of $A_{\mathrm{FB}}\left(q_{0}^{2}\right)=0$ arises through $H_{-}^{V} \simeq 0$ as can be seen from (D.1) which is due to cancellations between $C_{9}^{\text {eff }}$ and the photon pole term described by $C_{7}^{\text {eff }}$ in the lepton vector current. The LHCb collaboration has determined the location of the zero $q_{0}^{2}=4.9 \pm 0.9 \mathrm{GeV}^{2}[21]$ which is consistent with SM predictions. Naively, one might expect $\left|H_{0}\right|:\left|H_{-}\right|:\left|H_{+}\right| \simeq 1: \sqrt{2}: 0$ and $F_{L}\left(q_{0}^{2}\right) \simeq\left(1^{2}+1^{2}\right) /\left(1^{2}+1^{2}+\sqrt{2}^{2}\right)=1 / 2$. To get a realistic number one needs to take into account the effect of the prefactors of the polarisation vectors (A.4), which are related to the equivalence theorem (relevant to heavy Higgs boson physics for example e.g. [61]). The limit corresponds to $m_{B}^{2} \gg m_{K^{*}}^{2}, q^{2}$ with $q^{2}$ large enough such that the photon pole is not dominant. Formally this happens as the 0-helicity (longitudinal) polarisation tensor scales as $1 / m_{K^{*}}$ and $1 / \sqrt{q^{2}}$ (A.4) whereas the other directions do not encounter such an enhancement. The zero helicity component can therefore be enhanced by a factor $\zeta \equiv$ $\left(m_{B}^{2} / 2\right) /\left.\left(m_{K^{*}} \sqrt{q^{2}}\right)\right|_{q^{2} \simeq q_{0}^{2}} \simeq 7$ which leads to $F_{L}\left(q_{0}^{2}\right) \simeq \zeta\left(1^{2}+1^{2}\right) /\left(\zeta\left(1^{2}+1^{2}\right)+\sqrt{2}^{2}\right)=0.88$. This value is close to the maximum of $F_{L} \simeq 0.8$ found in the literature, e.g. [7]. Differences can be understood by the cancellation between $C_{7}^{\text {eff }}$ and $C_{9}^{\text {eff }}$ terms in $\left|H_{0}^{V}\right|$.

In summary $F_{L}$ can be expected to raise considerably from $1 / 3$ at the endpoint due to the equivalence theorem and then asymptotes to zero for low $q^{2}$ by virtue of the photon pole dominance. The effect of the zero of the $A_{\mathrm{FB}}$ enhances this effect and influences the maximum of $F_{L}$.

\section{E Tensor contributions to $d \Gamma / d \phi$ at the endpoint}

The quantity $r_{\phi}(3.5)$, in the dimension six basis, is given by [8]:

$$
r_{\phi}=-\frac{4}{9} \beta_{\ell}^{2} \frac{\left|H_{0}\right|^{2}+t_{1}}{\left(1+\frac{\beta_{\ell}^{2}}{3}\right)\left|H_{0}\right|^{2}+8 \frac{m_{\ell}^{2}}{q_{\max }^{2}} \operatorname{Re}\left(H_{0}^{L} H_{0}^{R *}\right)+t_{2}}, \quad\left|H_{0}\right|^{2} \equiv\left|H_{0}^{L}\right|^{2}+\left|H_{0}^{R}\right|^{2},
$$

where $\left|A_{0}\right|=\left|H_{0}\right|$ in the notation of [8]. The symbols $t_{1,2}$ stand for tensor operator contributions, which read as

$$
\begin{aligned}
& t_{1} \equiv-8\left(\left|H_{t 0}\right|^{2}+\left|H_{+-}\right|^{2}\right), \\
& t_{2} \equiv 4\left(4-8 / 3 \beta_{\ell}^{2}\right)\left|H_{t 0}\right|^{2}+16 / 3\left|H_{+-}\right|^{2}+16 \sqrt{2} m_{\ell} / \sqrt{q_{\max }^{2}} \operatorname{Re}\left(\left(H_{0}^{L}+H_{0}^{R}\right) H_{t 0}^{*}\right) .
\end{aligned}
$$


Here, we used $\left|H_{+-}\right|=\left|A_{\| \perp}\right|,\left|H_{t 0}\right|=\left|A_{t 0}\right|$ to translate from [8]. Possible differences in phase conventions of polarisation vector should cancel as they always appear in the combination $\omega(\lambda) \omega(\lambda)^{*}$.

Open Access. This article is distributed under the terms of the Creative Commons Attribution License (CC-BY 4.0), which permits any use, distribution and reproduction in any medium, provided the original author(s) and source are credited.

\section{References}

[1] M. Jacob and G.C. Wick, On the general theory of collisions for particles with spin, Annals Phys. 7 (1959) 404 [INSPIRE].

[2] H.E. Haber, Spin formalism and applications to new physics searches, hep-ph/9405376 [INSPIRE].

[3] R. Zwicky, Endpoint symmetries of helicity amplitudes, arXiv:1309.7802 [INSPIRE].

[4] B. Grinstein and D. Pirjol, Exclusive rare $B \rightarrow K^{*} \ell^{+} \ell^{-}$decays at low recoil: Controlling the long-distance effects, Phys. Rev. D 70 (2004) 114005 [hep-ph/0404250] [INSPIRE].

[5] B. Grinstein and D. Pirjol, Symmetry breaking corrections to heavy meson form-factor relations, Phys. Lett. B 533 (2002) 8 [hep-ph/0201298] [INSPIRE].

[6] N. Isgur and M.B. Wise, Relationship Between Form-factors in Semileptonic $\bar{B}$ and D Decays and Exclusive Rare $\bar{B}$ Meson Decays, Phys. Rev. D 42 (1990) 2388 [InSPIRE].

[7] C. Bobeth, G. Hiller and D. van Dyk, The Benefits of $\bar{B} \rightarrow \bar{K}^{*} l^{+} l^{-}$Decays at Low Recoil, JHEP 07 (2010) 098 [arXiv: 1006.5013] [INSPIRE].

[8] C. Bobeth, G. Hiller and D. van Dyk, General Analysis of $\bar{B} \rightarrow \bar{K}^{(*)} \ell^{+} \ell^{-}$Decays at Low Recoil, Phys. Rev. D 87 (2013) 034016 [arXiv:1212.2321] [INSPIRE].

[9] C. Hambrock, G. Hiller, S. Schacht and R. Zwicky, $B \rightarrow K^{*}$ Form Factors from Flavor Data to $Q C D$ and Back, arXiv:1308.4379 [InSPIRE].

[10] Particle Data Group collaboration, J. Beringer et al., Review of Particle Physics (RPP), Phys. Rev. D 86 (2012) 010001 [inSPIRE].

[11] M. Hopfer and R. Zwicky, in preparation, Edinburgh 2014-05.

[12] S. Weinberg, The Quantum theory of fields. Vol. 1: Foundations, Cambridge University Press, Cambridge, U.K. (1995).

[13] F. Krüger, L.M. Sehgal, N. Sinha and R. Sinha, Angular distribution and CP asymmetries in the decays $\bar{B} \rightarrow K^{-} \pi^{+} e^{-} e^{+}$and $\bar{B} \rightarrow \pi^{-} \pi^{+} e^{-} e^{+}$, Phys. Rev. D 61 (2000) 114028 [Erratum ibid. D 63 (2001) 019901] [hep-ph/9907386] [INSPIRE].

[14] C. Bobeth, G. Hiller and G. Piranishvili, CP Asymmetries in bar $B \rightarrow \bar{K}^{*}(\rightarrow \bar{K} \pi) \bar{\ell} \ell$ and Untagged $\bar{B}_{s}, B_{s} \rightarrow \phi\left(\rightarrow K^{+} K^{-}\right) \bar{\ell} \ell$ Decays at NLO, JHEP 07 (2008) 106 [arXiv:0805.2525] [INSPIRE].

[15] W. Altmannshofer, P. Ball, A. Bharucha, A.J. Buras, D.M. Straub et al., Symmetries and Asymmetries of $B \rightarrow K^{*} \mu^{+} \mu^{-}$Decays in the Standard Model and Beyond, JHEP 01 (2009) 019 [arXiv:0811.1214] [INSPIRE]. 
[16] S. Descotes-Genon, J. Matias, M. Ramon and J. Virto, Implications from clean observables for the binned analysis of $B->K^{*} \mu^{+} \mu^{-}$at large recoil, JHEP 01 (2013) 048 [arXiv: 1207.2753] [INSPIRE].

[17] F. Krüger and J. Matias, Probing new physics via the transverse amplitudes of $B 0 \rightarrow K^{* 0}\left(\rightarrow K^{-} \pi^{+}\right) \ell^{+} \ell^{-}$at large recoil, Phys. Rev. D 71 (2005) 094009 [hep-ph/0502060] [INSPIRE].

[18] C. Hambrock and G. Hiller, Extracting $B \rightarrow K^{*}$ Form Factors from Data, Phys. Rev. Lett. 109 (2012) 091802 [arXiv: 1204.4444] [INSPIRE].

[19] S. Akar [BaBar collaboration], Lake Louise Winter Institute, Canada, 23 February 2012, http://cds.cern.ch/record/1422422.

[20] CDF collaboration, Precise Measurements of Exclusive $b \rightarrow s \mu^{+} \mu^{-}$Decay Amplitudes Using the Full CDF Data Set, in proceedings of 36th International Conference for High Energy Physics (ICHEP), Melbourne, Australia, 4-11 July 2012 CDF note 10894.

[21] LHCb collaboration, Differential branching fraction and angular analysis of the decay $B^{0} \rightarrow K^{* 0} \mu^{+} \mu^{-}$, JHEP 08 (2013) 131 [arXiv:1304.6325] [INSPIRE].

[22] LHCb collaboration, Differential branching fraction and angular analysis of the decay $B_{s}^{0} \rightarrow \phi \mu^{+} \mu^{-}$, JHEP 07 (2013) 084 [arXiv: 1305.2168] [inSPIRE].

[23] LHCb collaboration, Measurement of form-factor independent observables in the decay $B^{0} \rightarrow K^{* 0} \mu^{+} \mu^{-}$, Phys. Rev. Lett. 111 (2013) 191801 [arXiv:1308.1707] [INSPIRE].

[24] ATLAS collaboration, Angular Analysis of $B_{d} \rightarrow K^{* 0} \mu^{+} \mu^{-}$with the ATLAS Experiment, ATLAS-CONF-2013-038 (2013).

[25] CMS collaboration, Angular analysis and branching fraction measurement of the decay $B^{0} \rightarrow K^{* 0} \mu^{+} \mu^{-}$, Phys. Lett. B 727 (2013) 77 [arXiv:1308.3409] [inSPIRE].

[26] W. Altmannshofer, A.J. Buras, D.M. Straub and M. Wick, New strategies for New Physics search in $B \rightarrow K^{*} \nu \bar{\nu}, B \rightarrow K \nu \bar{\nu}$ and $B \rightarrow X_{s} \nu \bar{\nu}$ decays, JHEP 04 (2009) 022 [arXiv: 0902.0160] [INSPIRE].

[27] Z. Ligeti and M.B. Wise, $|V(u b)|$ from exclusive B and D decays, Phys. Rev. D 53 (1996) 4937 [hep-ph/9512225] [INSPIRE].

[28] F. Krüger and L.M. Sehgal, Lepton polarization in the decays $b \rightarrow X(s) \mu^{+} \mu^{-}$and $B \rightarrow X(s) \tau^{+} \tau^{-}$, Phys. Lett. B 380 (1996) 199 [hep-ph/9603237] [INSPIRE].

[29] A. Ali, P. Ball, L.T. Handoko and G. Hiller, A Comparative study of the decays $B \rightarrow\left(K, K^{*}\right) \ell^{+} \ell^{-}$in standard model and supersymmetric theories, Phys. Rev. D 61 (2000) 074024 [hep-ph/9910221] [INSPIRE].

[30] LHCb collaboration, Observation of a resonance in $B^{+} \rightarrow K^{+} \mu^{+} \mu^{-}$decays at low recoil, Phys. Rev. Lett. 111 (2013) 112003 [arXiv:1307.7595] [INSPIRE].

[31] C. Bobeth, G. Hiller and D. van Dyk, More Benefits of Semileptonic Rare B Decays at Low Recoil: CP-violation, JHEP 07 (2011) 067 [arXiv: 1105.0376] [INSPIRE].

[32] M. Dimou, J. Lyon and R. Zwicky, Exclusive Chromomagnetism in heavy-to-light FCNCs, Phys. Rev. D 87 (2013) 074008 [arXiv: 1212.2242] [INSPIRE].

[33] P. Ball and R. Zwicky, $B(D, S) \rightarrow \rho, \omega, K^{*}, \phi$ decay form-factors from light-cone sum rules revisited, Phys. Rev. D 71 (2005) 014029 [hep-ph/0412079] [INSPIRE]. 
[34] R.R. Horgan, Z. Liu, S. Meinel and M. Wingate, Lattice QCD calculation of form factors describing the rare decays $B \rightarrow K^{*} \ell^{+} \ell^{-}$and $B_{s} \rightarrow \phi \ell^{+} \ell^{-}$, arXiv:1310.3722 [INSPIRE].

[35] P. Ball, G.W. Jones and R. Zwicky, $B \rightarrow V$ gamma beyond QCD factorisation, Phys. Rev. D 75 (2007) 054004 [hep-ph/0612081] [INSPIRE].

[36] LHCb collaboration, Measurement of $C P$ violation and the $B_{s}^{0}$ meson decay width difference with $B_{s}^{0} \rightarrow J / \psi K^{+} K^{-}$and $B_{s}^{0} \rightarrow J / \psi \pi^{+} \pi^{-}$decays, Phys. Rev. D 87 (2013) 112010 [arXiv: 1304.2600] [INSPIRE].

[37] BABAR collaboration, B. Aubert et al., Measurement of decay amplitudes of $B \rightarrow J / \psi K^{*}, \psi(2 S) K^{*}$ and $\chi_{c 1} K^{*}$ with an angular analysis, Phys. Rev. D 76 (2007) 031102 [arXiv: 0704.0522] [INSPIRE].

[38] LHCb collaboration, Measurement of the polarization amplitudes in $B^{0} \rightarrow J / \psi K^{*}(892)^{0}$ decays, Phys. Rev. D 88 (2013) 052002 [arXiv: 1307.2782] [inSPIRE].

[39] M. Beylich, G. Buchalla and T. Feldmann, Theory of $B \rightarrow K^{(*)} \ell^{+} \ell^{-}$decays at high $q^{2}$ : OPE and quark-hadron duality, Eur. Phys. J. C 71 (2011) 1635 [arXiv:1101.5118] [INSPIRE].

[40] C. Bobeth, G. Hiller and G. Piranishvili, Angular distributions of $\bar{B} \rightarrow$ Kll decays, JHEP 12 (2007) 040 [arXiv: 0709.4174] [INSPIRE].

[41] D. Becirevic and A. Tayduganov, Impact of $B \rightarrow K_{0}^{*} \ell^{+} \ell^{-}$on the New Physics search in $B \rightarrow K^{*} \ell^{+} \ell^{-}$decay, Nucl. Phys. B 868 (2013) 368 [arXiv:1207.4004] [InSPIRE].

[42] J. Lyon and R. Zwicky, Isospin asymmetries in $B \rightarrow\left(K^{*}, \rho\right) \gamma / l^{+} l^{-}$and $B \rightarrow K l^{+} l^{-}$in and beyond the Standard Model, Phys. Rev. D 88 (2013) 094004 [arXiv:1305.4797] [InSPIRE].

[43] R.-H. Li, C.-D. Lu and W. Wang, Branching ratios, forward-backward asymmetries and angular distributions of $B \rightarrow K_{2}^{*} l^{+} l^{-}$in the standard model and new physics scenarios, Phys. Rev. D 83 (2011) 034034 [arXiv:1012.2129] [INSPIRE].

[44] BABAR collaboration, B. Aubert et al., Observation and study of baryonic B decays: $B \rightarrow D(*) p \bar{p}, D(*) p \bar{p} \pi$ and $D(*) p \bar{p} \pi \pi$, arXiv:0908.2202 [INSPIRE].

[45] BeLLe collaboration, Y.-W. Chang et al., Observation of $B^{0} \rightarrow \Lambda \bar{\Lambda} K^{0}$ and $B^{0} \rightarrow \Lambda \bar{\Lambda} K^{* 0}$ at Belle, Phys. Rev. D 79 (2009) 052006 [arXiv:0811.3826] [inSPIRE].

[46] Belle collaboration, J.H. Chen et al., Observation of $B^{0} \rightarrow p \bar{p} K^{* 0}$ with a large $K^{* 0}$ polarization, Phys. Rev. Lett. 100 (2008) 251801 [arXiv:0802.0336] [INSPIRE].

[47] LHCb collaboration, Searches for $B_{(s)}^{0} \rightarrow J / \psi p \bar{p}$ and $B^{+} \rightarrow J / \psi p \bar{p} \pi^{+}$decays, JHEP 09 (2013) 006 [arXiv:1306.4489] [INSPIRE].

[48] A.L. Kagan, Polarization in $B \rightarrow V V$ decays, Phys. Lett. B 601 (2004) 151 [hep-ph/0405134] [INSPIRE].

[49] M. Beneke, J. Rohrer and D. Yang, Branching fractions, polarisation and asymmetries of $B \rightarrow V V$ decays, Nucl. Phys. B 774 (2007) 64 [hep-ph/0612290] [INSPIRE].

[50] LHCb collaboration, First measurement of the CP-violating phase in $B_{s}^{0} \rightarrow \phi \phi$ decays, Phys. Rev. Lett. 110 (2013) 241802 [arXiv: 1303.7125] [INSPIRE].

[51] LHCb collaboration, First observation of the decay $B_{s}^{0} \rightarrow \phi \bar{K}^{* 0}$, JHEP 11 (2013) 092 [arXiv:1306.2239] [INSPIRE].

[52] J. Matias, On the S-wave pollution of $B \rightarrow K^{*} \ell^{+} \ell^{-}$observables, Phys. Rev. D 86 (2012) 094024 [arXiv: 1209.1525] [INSPIRE]. 
[53] T. Blake, U. Egede and A. Shires, The effect of $S$-wave interference on the $B^{0} \rightarrow K^{* 0} \ell^{+} \ell^{-}$ angular observables, JHEP 03 (2013) 027 [arXiv: 1210.5279] [INSPIRE].

[54] S. Descotes-Genon, J. Matias and J. Virto, Understanding the $B \rightarrow K^{*} \mu^{+} \mu^{-}$Anomaly, Phys. Rev. D 88 (2013) 074002 [arXiv:1307.5683] [INSPIRE].

[55] W. Altmannshofer and D.M. Straub, New physics in $B \rightarrow K^{*} \mu \mu$ ?, Eur. Phys. J. C 73 (2013) 2646 [arXiv: 1308.1501] [INSPIRE].

[56] F. Beaujean, C. Bobeth and D. van Dyk, Comprehensive Bayesian Analysis of Rare (Semi)leptonic and Radiative B Decays, arXiv:1310.2478 [INSPIRE].

[57] C. Bobeth, G. Hiller, D. van Dyk and C. Wacker, The Decay $B \rightarrow K \ell^{+} \ell^{-}$at Low Hadronic Recoil and Model-Independent Delta B = 1 Constraints, JHEP 01 (2012) 107 [arXiv:1111.2558] [INSPIRE].

[58] U. Egede, T. Hurth, J. Matias, M. Ramon and W. Reece, New physics reach of the decay mode $\bar{B} \rightarrow \bar{K}^{* 0} \ell^{+} \ell^{-}$, JHEP 10 (2010) 056 [arXiv: 1005.0571] [InSPIRE].

[59] W.R. Reece, Exploiting angular correlations in the rare decay $B \rightarrow K^{*} \mu^{+} \mu^{-}$at LHCb, Ph.D. thesis, Imperial College, London, U.K. (2010) [http://cds.cern.ch/record/1280731].

[60] K. Petridis, Observables and phenomenology in $b \rightarrow$ sll decays, talk given at U.K. flavour workshop, Durham, United Kingdom, 4-7 September 2013 [http://conference.ippp.dur.ac.uk/ contributionDisplay.py? contribId $=32 \&$ sessionId $=6 \&$ confId $=338]$.

[61] J.F. Donoghue, E. Golowich and B.R. Holstein, Dynamics of the standard model, Cambridge Monographs on Particle Physics, Nuclear Physics and Cosmology 2 (1992) 1. 